$\underline{\text { Preprint typeset in JHEP style. - HYPER VERSION }}$

OUTP-00-31P

hep-th/0012188

\title{
Open and Unoriented Strings from Topological Membrane I. Prolegomena
}

\author{
P. Castelo Ferreira* \\ Department of Physics - Theoretical Physics, University of Oxford \\ 1 Keble Road, Oxford OX1 3NP, U.K. \\ Ian I. Kogan ${ }^{\dagger}$ \\ Department of Physics - Theoretical Physics, University of Oxford \\ 1 Keble Road, Oxford OX1 3NP, U.K.
}

\begin{abstract}
We study open and unoriented strings in a Topological Membrane (TM) theory through orbifolds of the bulk $3 D$ space. This is achieved by gauging discrete symmetries of the theory. Open and unoriented strings can be obtained from all possible realizations of $C, P$ and $T$ symmetries. The important role of $C$ symmetry to distinguish between Dirichlet and Neumman boundary conditions is discussed in detail.
\end{abstract}

KEYwords: TM(GT), Strings, CFT, orbifold, Chern-Simons.

*e-mail: pcastelo@thphys.ox.ac.uk

†e-mail: kogan@thphys.ox.ac.uk 


\section{Contents}

1. Introduction 2

2. Riemann Surfaces:

from Closed Oriented to Open and Unoriented

2.1 The Projective Plane and the Disk obtained from the Sphere 5

2.2 The annulus, Möbius strip and Klein bottle from the Torus 8

3. Conformal Field Theory -

Correlation Functions and Boundary Conditions 11

3.1 Disk 11

3.1.1 Dirichlet Boundary Conditions 12

3.1.2 Neumann Boundary Conditions 13

3.2 Annulus 14

3.2.1 Dirichlet Boundary Conditions 14

3.2.2 Neumann Boundary Conditions 15

4. $\operatorname{TM}(\mathrm{GT})$

4.1 Horava Approach to Open World-Sheets

4.2 Discrete Symmetries and Orbifold of TM(GT) 19

4.3 Tree Level Amplitudes for

Open and Closed Unoriented Strings 22

4.3.1 Disk 23

4.3 .2 Projective Plane 27

4.4 One Loop Amplitudes for

Open and Closed Unoriented Strings

4.4.1 Annulus 30

4.4 .2 Möbius Strip 31

4.4 .3 Klein Bottle 33

4.5 Note on Modular Invariance and the Relative Modular Group 33

4.6 Neumann and Dirichlet World-Sheet Boundary Conditions,

Monopoles Processes and Charge Conjugation 36

4.7 T-Duality and Several U(1)'s 38

5. Conclusion and Discussion 39 


\section{Introduction}

Although originally (and historically) open string theories were considered as theories by themselves, it soon become evident that, whenever they are present, they come along with closed (non-chiral) strings. Moreover open string theories are obtained from closed string theories by gauging certain symmetries of the closed theory (see [1] and references therein for a discussion of this topic). The way to get open strings from closed strings is by gauging the world-sheet parity [1-3], $\Omega: z \rightarrow-\bar{z}$. That is we impose the identification $\sigma_{2} \cong-\sigma_{2}$, where $z=\sigma_{1}+i \sigma_{2}$ and $\bar{z}=\sigma_{1}-i \sigma_{2}$ ) is the complex structure of the world-sheet manifold. The spaces obtained in this way can be of two types: closed unoriented and open oriented (and unoriented as well). These last ones are generally called orbifolds and the singular points of the construction become boundaries. The states (operators and fields of the theory in general) of the open/unoriented theory are obtained from the closed oriented theory by projecting out the ones which have negative eigenvalues of the parity operator. This is obtained by building a suitable projection operator $(1+\Omega) / 2$ such that only the states of positive eigenvalues are kept in the theory. Namely the identification $X^{I}(z, \bar{z}) \cong X^{I}(\bar{z}, z)$ or $X_{L}^{I}(z) \cong X_{R}^{I}(\bar{z})$ (in terms of the holomorphic and antiholomorphic parts of $X=X_{L}+X_{R}$ ) holds.

Another construction in string theory is orbifolding the target space of the theory under an involution of some symmetry of that space. In this work we are going to consider only a $Z_{2}$ involution, imposing the identification $X^{I} \cong-X^{I}$, where $X^{I}$ are the target space coordinates. When combining both constructions, world-sheet and target space orbifolding, we obtain open/unoriented theories in orbifolds [4-7] or orientifolds $\left(X^{I}(z, \bar{z})=-X^{I}(\bar{z}, z)\right)$, implying the existence of twisted sectors in the open/unoriented theories.

Further to the previous discussion both sectors (twisted and untwisted) need to be present for each surface in order to ensure modular invariance of the full partition function $[1,8,18]$. One point we want to stress is that twisting in open strings can, for the case of a $Z_{2}$ target space orbifold, be simply interpreted as the choice of boundary conditions: Neumman or Dirichlet.

Toroidal compactification is an important construction in string theories and in the web of target space dualities. Early works considered also open string constructions in these toroidal backgrounds $[8,9]$. In these cases we have some compactified target space coordinates, say $X^{J}(z+2 \pi i, \bar{z}-2 \pi i) \cong X^{J}(z, \bar{z})+2 \pi R(R$ is the radius of compactification of $\left.X^{J}\right)$, the twisted states in the theory are the ones corresponding to the points identified under $X^{J}(z+2 \pi i, \bar{z}-2 \pi i) \cong-X^{J}(z, \bar{z})+2 \pi R$ or in terms of the holomorphic and antiholomorphic parts of $X$ this simply reads $X_{L}^{I}(z) \cong-X_{R}^{I}(\bar{z})$.

An important result coming from these constructions is that the gauge group of the open theory, the Chan-Paton degrees of freedom carried by the target space photon Wilson lines (only present in open theories) are constrained, both due to 
dualities of open string theory [8] and to modular invariance of open and unoriented theories [8-11]. This will result in the choice of the correct gauge group that cancels the anomalies in the theory.

One fundamental ingredient of string theory is modular invariance. Although for bosonic string theory the constraints coming from genus 1 amplitudes are enough to ensure modular invariance at generic genus $g$, it becomes clear that once the fermionic sector of superstring theory is considered it is necessary to consider genus 2 amplitude constraints. For closed strings (types II and 0) the modular group at genus $g$ is $S P L(2 g, \mathbb{Z})$ and the constraints imposed by modular invariance at $g=2$ induce several possible projections in the state space of the theory [12-16] such that the resulting string theories are consistent. Among them are the well known GSO projections [17] that insure the correct spin-statistics connection, project out the tachyon and ensure a supersymmetric effective theory in the $10 D$ target space.

Once we consider an open superstring theory (type I) created by orbifolding the world-sheet parities, for each open (and/or unoriented) surface a Relative Modular Group still survives the orbifold at each genus $g$ [18]. Again in a similarly way to the closed theory the modular invariance under these groups will result in generalized GSO projections [18-21].

For a more recent overview of the previous topics see $[22,23]$ (see also [24] for an extensive explanation of them).

The purpose of this work is to build open, open unoriented, and closed unoriented string theories (with and without orbifolding of the target space) from the Topological Membrane (TM) [25-40]. This approach consists of a Topological Massive Gauge Theory (TMGT) [41-43] living on a 3D membrane, i.e. a Maxwell term and a gauge Chern-Simons term, together with Topological Massive Gravity (TMG), i.e. Einstein and a gravitational Chern-Simons term. The membrane is a $3 D$ manifold $M=\Sigma \times[0,1]$ which has two boundaries $\partial M=\Sigma_{0}+\Sigma_{1}$. Gauge transformations induce chiral Conformal Field Theories on the boundaries. The first works were concerning only $3 D$ pure Chern-Simons theories in the bulk [44-46].

Closed string theories are obtained as the effective boundary theory, their worldsheet is the closed boundary $\partial M$. Obtaining open string theory raises a problem, we need a open world-sheet to define them. But the boundary of a boundary is zero, $\partial \partial M=0$. So naively it seems that TM cannot describe open strings since world-sheets are already a boundary of a $3 D$ manifold. The way out is to consider orbifolding of the bulk theory. In this way the fixed points of the orbifold play the role of the boundary of the $2 D$ boundary of the $3 D$ membrane. This proposal was first introduced by Horava [47] in the context of pure Chern-Simons theories. We are going to extend his results to TMGT and reinterpret the orbifolded group as symmetries of the full gauge theory.

Other works have developed Horava's idea. For a recent study on WZNW orbifold constructions see [48] (and references therein) For an extensive study, although 
in a more formal way than our work, of generic Rational Conformal Field Theories (RCFT) with boundaries from pure 3D Chern-Simons theory see [49] (and references therein). Nevertheless previously the monopole processes were not studied. These are crucial for describing the winding modes and T-duality in compact RCFT from the TM point of view and, therefore, in compactified string theories.

We consider an orbifold of $\mathrm{TM}(\mathrm{GT})$ such that one new boundary is created at the orbifold fixed point. To do this we gauge the discrete symmetries of the $3 D$ theory, namely $P T$ and $P C T$. Several $P$ 's are going to be defined as generalized parity operations. $C$ and $T$ are the usual $3 D$ QFT charge conjugation and time inversion operations (see [50] for a review). The orbifolding of the string target space corresponds in pure Chern-Simons membrane theory to the quotient of the gauge group by a $Z_{2}$ symmetry [45]. As will be shown, in the full $\mathrm{TM}(\mathrm{GT})$, the discrete symmetry which will be crucial in this construction is charge conjugation $C$. Besides selecting between twisted and untwisted sectors in closed unoriented string theory it will also be responsible for setting Neumann and Dirichlet boundary conditions in open string theory. In this work we are not going to consider more generic orbifold groups.

There are two main new ideas introduced in this work. Firstly the use of all possible realizations of $P, C$ and $T$ combinations, which constitute discrete symmetries of the theory, as the orbifold group. Although the mechanism is similar to the one previously studied by Horava for pure Chern-Simons theory, the presence of the Maxwell term constrains the possible symmetries to PT and PCT type only. Also the interpretation of the orbifold group as the discrete symmetries in the quantum theory is new, as is the interpretation of charge conjugation $C$ which selects between Neumman and Dirichlet boundary conditions. This symmetry explains the T-duality of open strings in the TM framework. It is a symmetry of the $3 D$ bulk which exchanges trivial topological configurations (without monopoles) with non-trivial topological configurations (with monopoles). In terms of the effective boundary CFT (string theory) this means exchanging Kaluza-Klein modes (no monopole effects in the bulk) with winding number (monopole effects in the bulk).

In section 2 we start by introducing genus 0 (the sphere), and genus 1 (the torus), Riemann surfaces and their possible orbifolds under discrete symmetries which we identify with generalized parities $P$.

Section 3 gives an account of Neumann and Dirichlet boundary conditions in usual CFT using the Cardy method [51] of relating $n$ point full correlation functions in boundary Conformal Field Theory with $2 n$ chiral correlation functions in the theory without boundaries.

Then, in section 1 we give a brief overview of the discrete symmetries of $3 D$ QFT and use it to orbifold $\mathrm{TM}(\mathrm{GT})$. We enumerate the $3 D$ configurations compatible with the several orbifolds, both at the level of the field configurations and of the particular charge spectrums corresponding to the resulting theories. It naturally emerges 
from the $3 D$ membrane that the configurations compatible with $P C T$ correspond to Neumann boundary conditions (for open strings) and to untwisted sectors (for closed unoriented). The configurations compatible with PT correspond to Dirichlet boundary conditions (for open strings) and twisted sectors (for closed unoriented). The genus 2 constraints are discussed here although a more detailed treatment is postponed for future work. Further it is shown that Neumann (untwisted) corresponds to the absence of monopole induced processes while for Dirichlet (twisted) these processes play a fundamental role. A short discussion on T-duality show that it has the same bulk meaning as modular invariance, they both exchange $P T \leftrightarrow P C T$.

\section{Riemann Surfaces: from Closed Oriented to Open and Unoriented}

Any open or unoriented manifold $\Sigma_{u}$ can, in general, be obtained from some closed orientable manifolds $\Sigma$ under identification of a $Z_{2}$ (or at most two $Z_{2}$ ) involution

$$
\begin{aligned}
\pi: \quad \Sigma & \rightarrow \Sigma_{u}=\Sigma / Z_{2} \\
(x,-x) & \rightarrow x
\end{aligned}
$$

such that each point in $\Sigma_{u}$ has exactly two corresponding points in $\Sigma$ conjugate in relation to the $Z_{2}$ involution(s). The pair $(x,-x)$ in the last equation is symbolic, the second element stands for the action of the group $Z_{2}, z_{2}(x)=-x$, in the manifold. Usually this operation is closely related with parity as will be explained bellow. Although in this work our perspective is that we start from a full closed oriented theory and orbifold it, there is the reverse way of explaining things. This means that any theory defined in an open/unoriented manifold is equivalently defined in the closed/oriented manifold which doubles (consisting of two copies of) the original open/unoriented.

Let us summarize how to obtain the disk $D_{2}$ (open orientable) and projective plane $R P_{2}$ (closed unorientable) out of the sphere $S^{2}$ and the annulus $C_{2}$ (open orientable), the Möbius Strip (open unorientable) and Klein bottle $K_{2}$ (closed unorientable) out of the torus $T^{2}$.

\subsection{The Projective Plane and the Disk obtained from the Sphere}

For simplicity we choose to work in complex stereographic coordinates $\left(z=x_{1}+i x_{2}\right.$, $\left.\bar{z}=x_{1}-i x_{2}\right)$ such that the sphere is identified with the full complex plane. The sphere has no moduli and the Conformal Killing Group $(\mathrm{CKG})$ is $P S L(2, \mathbb{C})$. A generic element of this group is $(a, b, c, d)$ with the restriction $a d-b c=1$. It acts in a point as

$$
z^{\prime}=\frac{a z+b}{c z+d}
$$



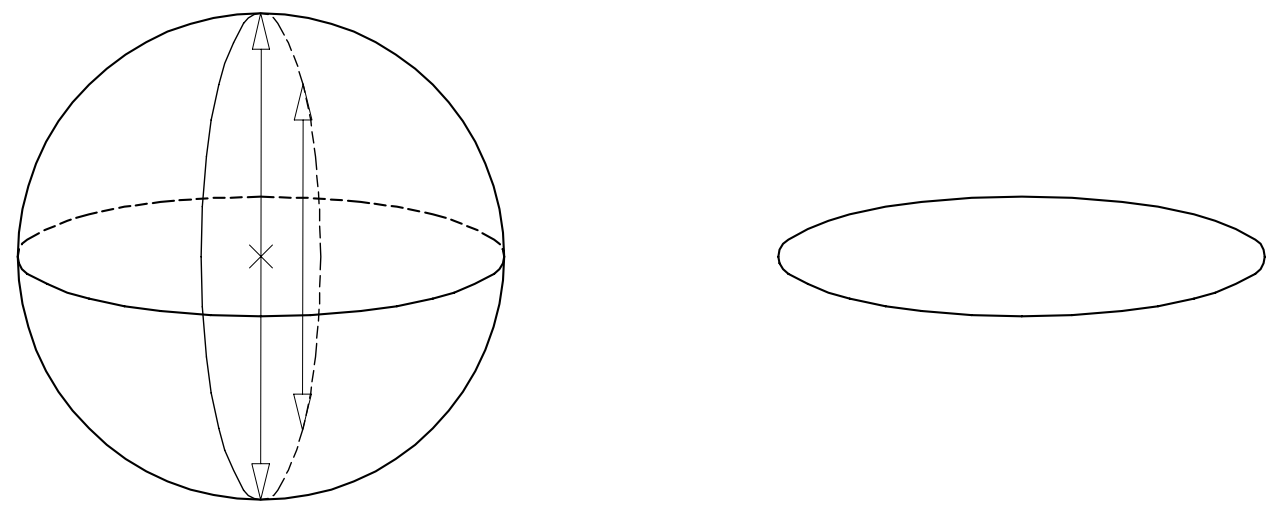

Figure 1: The disk $D_{2}=S^{2} / P_{1}$ obtained from the sphere under the involution given by the parity operation $P_{1}$.

It has then six real parameters, that is, six generators. That is to say that the sphere has six Conformal Killing Vectors (CKV's). It is necessary to use two coordinate charts to cover the full sphere, one including the north pole and the other one including the south pole. Usually it is enough to analyze the theory defined on the sphere only for one of the patches but it is necessary to check that the transformation between the two charts is well defined. In stereographic complex coordinates the map between the two charts (with coordinates $z, \bar{z}$ and $u, \bar{u}$ ) is given by $z \rightarrow 1 / u$ and $\bar{z} \rightarrow 1 / \bar{u}$.

The disk $D_{2}$ can be obtained from the sphere under the identification

$$
z \cong \bar{z}
$$

This result is graphically pictured in figure 1 and consists in the involution of the manifold $S^{2}$ by the group $Z^{P_{1}}, D_{2}=S^{2} / Z^{P_{1}}$. There are one boundary corresponding to the real line in the complex plane and the disk is identified with the upper half complex plane.

It is straightforward to see that the non trivial element of $Z^{P_{1}}$ is nothing else than the usual $2 D$ parity transformation

$$
\begin{aligned}
P_{1}: z & \rightarrow \bar{z} \\
\bar{z} & \rightarrow z
\end{aligned}
$$

The CKG of the disk is the subgroup of $P S L(2, \mathbb{C})$ which maintains constraint (2.3), that is $\operatorname{PSL}(2, \mathbb{R})$.

From the point of view of the fields defined in the sphere this corresponds to the usual $2 D$ parity transformation. In order that the theory be well defined in the orbifolded sphere we have to demand the fields of the theory to be compatible with the construction

$$
\begin{aligned}
f(z) & =f\left(P_{1}(z)\right) \\
\phi_{i}\left[x_{j}\right] & =P_{1} \phi_{i}\left[P_{1}\left(x_{j}\right)\right]
\end{aligned}
$$



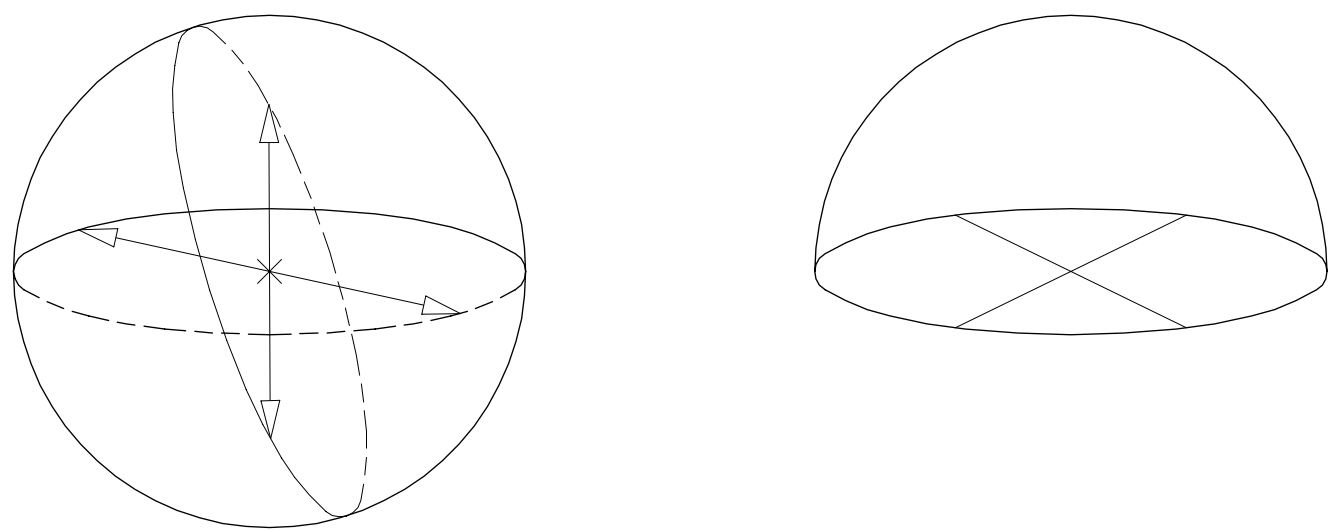

Figure 2: The projective plane $R P_{2}=S^{2} / P_{2}$ obtained from the sphere under the involution given by the parity operation $P_{2}$.

where the first equation applies to scalar fields and the second to vectorial ones. For tensors of generic dimensions $d$ (e.g. the metric or the antisymmetric tensor) the transformation is easily generalized to be $T(x)=P_{1}^{d} T\left(P_{1}(x)\right)$.

In order to orbifold the theory defined on the sphere we can introduce the projection operator

$$
P_{1, \text { proj }}=\frac{1}{2}\left(1+P_{1}\right)
$$

which projects out every operator with odd parity eigenvalue and keeps in the theory only field configurations compatible with the $Z_{2}$ involution.

To obtain the projective plane $R P_{2}$ we need to make the identification

$$
z \cong-\frac{1}{\bar{z}}
$$

This result is graphically pictured in figure 2 and again is an involution of the sphere $R P_{2}=S^{2} / Z_{2}^{P_{2}}$. The resulting space has no boundary and no singular points. But it is now an unoriented manifold.

This identification can be thought of as two operations. The action of the element $\alpha=(0,-1,1,0) \in Z_{2}^{\alpha} \subset S L(2, \mathbb{C})$ followed by the operation of parity as given by (2.4). Note that $\alpha(z)=-1 / z$ but $P_{1} \alpha(z)=-1 / \bar{z}$ as desired. In this case we can define a new parity operation $P_{2} \in Z_{2}^{P_{2}}=Z_{2}^{P_{1}} \times Z_{2}^{\alpha}$ as

$$
\begin{aligned}
P_{2}: z & \rightarrow-\frac{1}{\bar{z}} \\
\bar{z} & \rightarrow-\frac{1}{z}
\end{aligned}
$$

From the point of view of the fields defined in the sphere we could use the usual parity transformation since any theory defined in the sphere should be already invariant under transformation (2.2) such that $\operatorname{PSL}(2, \mathbb{C})$ is a symmetry of the theory. But 
in order to have a more transparent picture we use the definition (2.8) of $P_{2}$ and demand that

$$
\begin{aligned}
f(z) & =f\left(P_{2}(z)\right) \\
\phi_{i}\left[x_{j}\right] & =P_{2}\left(\phi_{i}\left[P_{2}\left(x_{j}\right)\right]\right)
\end{aligned}
$$

where the first equation concerns to scalar fields and the second to vectorial ones. For tensors of generic dimensions $d$ (as the metric or the antisymmetric tensor) the transformation is again easily generalized to be $T(x)=P_{2}^{d} T\left(P_{2}(x)\right)$.

The CKG is now $S O(3)$, the usual rotation group. It is the subgroup of $P S L(2, \mathbb{C}) / Z_{2}^{\alpha}$ that maintains constraint (2.3)

\subsection{The annulus, Möbius strip and Klein bottle from the Torus}

Let us proceed to genus one closed orientable manifold, the torus. It is obtained from the complex plane under the identifications

$$
z \cong z+2 \pi \cong z+2 \pi\left(\tau_{1}+i \tau_{2}\right)
$$

There are two modular parameters $\tau=\tau_{1}+i \tau_{2}$ and two CKV's. The action of the $\mathrm{CKG}$, the translation group in the complex plane, is

$$
z^{\prime}=z+a+i b
$$

with $a$ and $b$ real. The metric is simply $\left|d x^{1}+\tau d x^{2}\right|$ and the identifications on the complex plane are invariant under the two operations

$$
\mathcal{T}: \tau^{\prime}=\tau+1 \quad \mathcal{S}: \tau^{\prime}=-\frac{1}{\tau}
$$

These operations constitute the modular group $P S L(2, \mathbb{Z})$. That is

$$
\tau^{\prime}=\frac{a \tau+b}{c \tau+d}
$$

with $a, b, c, d \in \mathbb{Z}$ and $a d-b c=1$.

The annulus $C_{2}$ (or topologically equivalent, the cylinder) is obtained from the torus with $\tau=i \tau_{2}$ under the identification

$$
z \cong-\bar{z}
$$

This result is symbolically picture in figure 3 .

There is now one modular parameter $\tau_{2}$ and no modular group. There is only one CKV being the CKG action given by $z^{\prime}=z+i b$, translation in the imaginary direction. In terms of the fields defined in the torus this correspond to the projection under the parity operation

$$
\begin{array}{r}
\Omega: z \rightarrow-\bar{z} \\
\bar{z} \rightarrow-z
\end{array}
$$



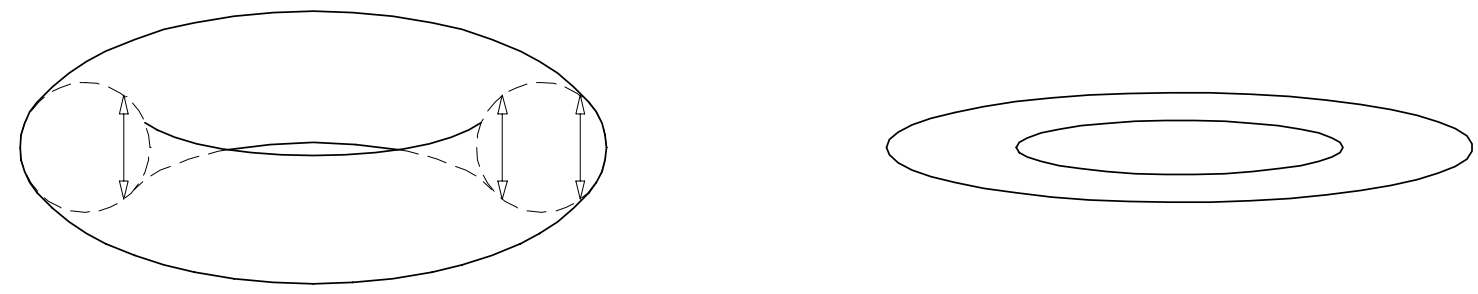

Figure 3: The annulus (or cylinder) $C_{2}=T^{2} / \Omega$ obtained from the torus under the involution given by the parity operation $\Omega$.

The Möbius strip $M_{2}$ can be obtained from the annulus (obtained from the torus with $\tau=2 i \tau_{2}$ ) by the identification under the element $\tilde{a}$ [24] of the translation group

$$
\tilde{a}: z \rightarrow z+2 \pi\left(\frac{1}{2}+i \tau_{2}\right)
$$

Note that $\tilde{a}$ belongs to the translation group of the torus, not of the disk, and that $\tilde{a}^{2}=1$. This construction corresponds to two involutions, so the orbifolding group is constituted by two $Z_{2}$ 's, $M_{2}=T^{2} /\left(Z_{2}^{\Omega} \otimes Z_{2}^{\tilde{a}}\right)$, where $\otimes$ stands for the semidirect product of groups. Thus the ratio of areas between the Möbius strip and the original torus is $1 / 4$ contrary to the $1 / 2$ of the remaining open/unoriented surfaces obtained from the torus, due to the extra projection operator $(1+\tilde{a}) / 2$ taking from the annulus to the strip.

In terms of the fields living on the torus we can think of this identification as the projection under a new discrete symmetry, which we also call parity

$$
\tilde{\Omega} \equiv \tilde{a} \circ \Omega
$$

Although this operation does not seem to be a conventional parity operation note that, applying it twice to some point, we retrieve the same point, $\tilde{\Omega}^{2}=1$. It is in this sense a generalized parity operation.
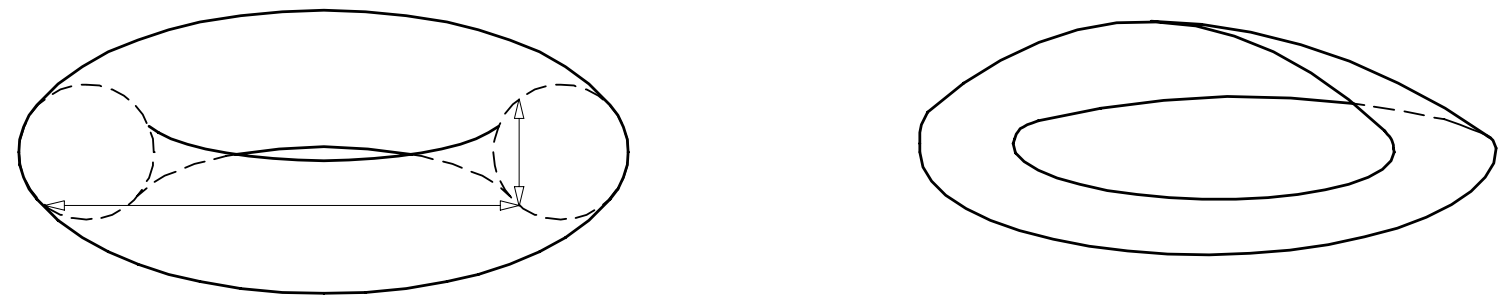

Figure 4: The Möbius strip $M_{2}=T^{2} / \tilde{\Omega}$ obtained from the torus under the involution given by the parity operation $\tilde{\Omega}$.

The previous construction is presented, for example, in Polchinski's book [24]. Let us note however that one can build the Möbius strip directly from a torus [1] 
with moduli $\tau=1 / 2+i \tau_{2}$ under the involution by $\Omega$ as given in (2.15) ${ }^{3}$. In this case the ratio of areas between the original torus and the involuted surface is $1 / 2$ as the other involutions studied in this section. As we will show later both constructions correspond to the same region on the complex plane. The first one results from two involutions of a torus $(\tau=2 i \tau)$ with double the area of the second construction $(\tau=i \tau)$. In this sense both constructions are equivalent. The Möbius strip orbifolding is pictured in figure 4 .

Again there is one modular parameter $\tau_{2}$ and no modular group. The only CKV is again the translation in the imaginary direction.

The Klein bottle $K_{2}$ is obtained from the torus with $\tau=2 i \tau_{2}$ under the identification

$$
z \cong-\bar{z}+2 \pi i \tau_{2}
$$

This result is pictured in figure 5 .
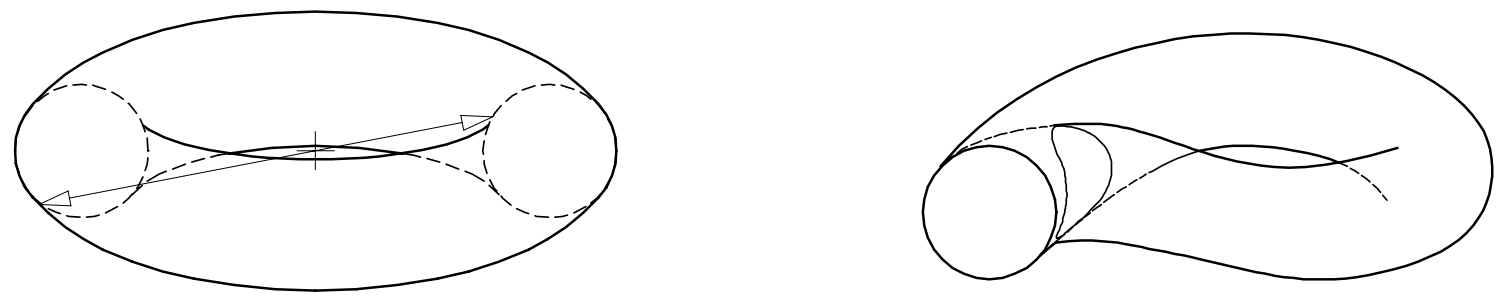

Figure 5: The Klein bottle $K_{2}=T^{2} / \Omega^{\prime}$ obtained from the torus under the involution given by the parity operation $\Omega^{\prime}$.

\begin{tabular}{|ccc|cccc|}
\hline $\mathbf{S}_{\mathbf{2}}$ & $P_{1}$ & $P_{2}$ & $\mathbf{T}_{\mathbf{2}}$ & $\Omega$ & $\Omega$ & $\Omega^{\prime}$ \\
& & & & & & \\
& $z \leftrightarrow \bar{z}$ & $z \leftrightarrow-1 / \bar{z}$ & & $z \leftrightarrow-\bar{z}$ & $\tilde{a} \circ \Omega$ & $z \rightarrow-\bar{z}+2 \pi i \tau_{2}$ \\
& & & & & & $\bar{z} \rightarrow-z+2 \pi i \tau_{2}$ \\
$S^{2} / P=$ & $D_{2}$ & $R P_{2}$ & $T^{2} / P=$ & $C_{2}$ & $M_{2}$ & $K_{2}$ \\
$\mathrm{C} / \mathrm{O}$ & $\mathrm{O} / \mathrm{O}$ & $\mathrm{C} / \mathrm{U}$ & $\mathrm{C} / \mathrm{O}$ & $\mathrm{O} / \mathrm{O}$ & $\mathrm{O} / \mathrm{U}$ & $\mathrm{C} / \mathrm{U}$ \\
& & & & & & \\
\hline
\end{tabular}

Table 1: Parity operations for the topology $T^{2} \times[0,1]$. The torus geometry considered is $\tau=i \tau_{2}$ for $\Omega$ and $\tau=2 i \tau_{2}$ for $\tilde{\Omega}$ and $\Omega^{\prime}$. Note that $M_{2}$ can also be obtained from the torus with $\tau=1 / 2+i \tau_{2}$ considering the parity $\Omega$. In the labels of the last line the first letter stands for Open or Close surface while the second letter stands for Oriented or Unoriented.

The bottle is the involution of the torus $K_{2}=T^{2} / Z_{2}^{\Omega^{\prime}}$, has one parameter CKG with one $\mathrm{CKV}$, translations in the imaginary direction. There is one modulus $\tau_{2}$ and

\footnotetext{
${ }^{3}$ The authors thank the referee for this useful remark!
} 
no modular group. The resulting manifold has no boundary and no singular points but is unoriented.

Again we can define a new parity transformation $\Omega^{\prime}$

$$
\begin{aligned}
\Omega^{\prime}: z & \rightarrow-\bar{z}+2 \pi i \tau_{2} \\
\bar{z} & \rightarrow-z+2 \pi i \tau_{2}
\end{aligned}
$$

We summarize in table 1 all the parity operations we have just studied together with the resulting involutions (or orbifolds).

\section{Conformal Field Theory - Correlation Functions and Boundary Conditions}

To study string theory we need to know the world-sheet CFT. In a closed string theory they are given by CFT on a closed Riemann surface, the simplest of them is the sphere, or equivalently the complex plane. To study open strings we need to study CFT on open surfaces. As was shown by Cardy [51] n-point correlation functions on a surface with a boundary are in one-to-one correspondence with chiral $2 n$ point correlation functions on the double surface ${ }^{4}$ (for more details and references see [52]).

We will study the disk and the annulus, so we double the number of charges (vertex operators) by inserting charges $\pm q$ (vertex operators with $\Delta=2 q^{2} / k$ ) in the Parity conjugate points. Note that the sign of the charges inserted depends on the type of boundary conditions that we want to impose but the conformal dimension of the corresponding vertex operator is the same.

We summarize the 2, 3 and 4-point holomorphic correlation functions of vertex operators for the free boson

$$
\begin{aligned}
<\phi\left(z_{1}\right) \phi\left(z_{2}\right) & >=z_{12}^{-2 \Delta} \\
<\phi(z 1) \phi(z 2) \phi(z 3)> & =z_{12}^{-\Delta_{1}-\Delta_{2}+\Delta_{3}} z_{13}^{-\Delta_{1}+\Delta_{2}-\Delta_{3}} z_{23}^{\Delta_{1}-\Delta_{2}-\Delta_{3}} \\
<\phi\left(z_{1}\right) \phi\left(z_{2}\right) \phi\left(z_{3}\right) \phi\left(z_{4}\right) & >=\prod_{i<j} z_{i j}^{2 q_{i} q_{j} / k}
\end{aligned}
$$

where in all the cases $\sum q_{i}=0$, otherwise they vanish.

\subsection{Disk}

We will take the disk as the upper half complex plane. As explained before it is obtained from the sphere (the full complex plane) by identifying each point in the

\footnotetext{
${ }^{4}$ One of the constructions presented to obtain the Möbius strip uses the double involution under $\tilde{\Omega}$. In that case $n$ insertions on it correspond to $4 n$ in the original torus.
} 
lower half complex plane with it's conjugate in the upper half complex plane. In terms of correlation functions

$$
\left\langle\phi_{q}(x, y)\right\rangle_{D_{2}}=\left\langle\phi_{q}(z) \phi_{-q}(\bar{z})\right\rangle_{S^{2}}
$$

where we replaced $z=x+i y$ in the the first equation of (3.1), $y$ is the distance to the real axis while $x$ is taken to be the horizontal distance (parallel to the real axis) between vertex insertions.

\subsubsection{Dirichlet Boundary Conditions}

As it is going to be shown, when the mirror charge have opposite sign the boundary conditions are Dirichlet.

The 2-point correlation function restricted to the upper half plane is simply the expectation value

$$
\left\langle\phi_{q}(x, y)\right\rangle=\frac{1}{(2 y)^{2 \Delta}}
$$

Insertion of vertex operators (from the unity) in the boundary is not compatible with the boundary conditions since the only charge that can exist there is $q=0$ (since $q=-q=0$ in the boundary). Taking the limit $y \rightarrow 0$ the expectation value (3.3) blows up but this should not worry us, near the boundary the two charges annihilate each other. This phenomena is nothing else than the physical counterpart of the operator fusion rules $\phi_{q}(y) \phi_{-q}(-y) \rightarrow(2 y)^{-2 \Delta} \phi_{0}(y)$. That is $\left\langle\phi_{0}\right\rangle_{\partial D_{2}}=\langle 1\rangle_{\partial D_{2}}$ in the boundary of the disk.

3-point correlation functions cannot be used for the same reason, one of the insertions would need to lie in the boundary but that would mean $q_{3}=0$, the other two charges had to be inserted symmetrically in relation to the real axis and would imply $q_{1}=-q_{2}$. This reduces the 3-point correlator to a 2-point one in the full plane.

For 4-point vertex insertions consider $q_{1}$ and $q_{3}$ in the upper half plane, $q_{2}$ (inserted symmetrically to $q_{1}$ ) and $q_{4}$ (inserted symmetrically to $q_{3}$ ) in the lower half plane. As pictured in figure 6 the most generic configurations is $q_{1}=-q_{2}=q$ and $q_{3}=-q_{4}=q^{\prime}$. Making $z_{2}=\bar{z}_{1}=-i y$ and $z_{4}=\bar{z}_{3}=x-i y^{\prime}$ we obtain the corresponding 2-point correlators in the upper half plane

$$
\left\langle\phi_{q}(0, y) \phi_{q^{\prime}}\left(x^{\prime}, y^{\prime}\right)\right\rangle=\frac{1}{(2 y)^{2 \Delta}\left(2 y^{\prime}\right)^{2 \Delta^{\prime}}}\left(1-\frac{4 y y^{\prime}}{x^{2}+\left(y+y^{\prime}\right)^{2}}\right)^{\frac{2 q q^{\prime}}{k}}
$$

Again note that we cannot insert boundary operators without changing the boundary conditions. In the limit $x \rightarrow \infty$ both correlators behave like

$$
\lim _{x \rightarrow \infty}\left\langle\phi\left(y_{1}\right) \phi\left(y_{2}\right)\right\rangle=\frac{1}{\left(4 y_{1} y_{2}\right)^{2 \Delta}}
$$

When we approach the boundary the correlators go to infinite independently of the value of $x$. This fact can be explained by the kind of boundary conditions we are 
considering, they are such that when the fields approach the boundary they become infinitely correlated independently of how far they are from each other. Therefore this must be Dirichlet boundary conditions, the fields are fixed along the boundary, furthermore, as stated before their expectation value is $\langle 1\rangle$. It doesn't mater how much apart they are, they are always correlated on the boundary. The tangential derivative to the boundary of the expectation value $\left.\partial_{x}\langle\phi\rangle\right|_{\partial D_{2}}=0$ also agrees with Dirichlet boundary conditions.

\subsubsection{Neumann Boundary Conditions}

For the case of the mirror charge having the same sign of the original one the boundary conditions will be Neumann. The expectation value for the fields in the bulk vanishes since the 2-point function $\left\langle\phi_{q}\left(z_{1}\right) \phi_{q}\left(z_{2}\right)\right\rangle=0$ in the full plane. Nevertheless we can evaluate directly the non-zero 2-point correlation function in the boundary

$$
\left\langle\phi_{q}(0) \phi_{-q}(x)\right\rangle=\frac{1}{x^{2 \Delta}}
$$

Note that contrary to the previous discussion, concerning Dirichlet boundary conditions, in this case $q \neq 0$ on the boundary since the mirror charges have the same sign and the correlation function vanishes in the limit $x \rightarrow \infty$ indicating that the boundary fields become uncorrelated.

The 3-point correlation function in the full plane must be considered with one charge $-2 q$ in the boundary and two other charges $q$ inserted symmetrically in relation to the real axis (see figure 6). In the upper half plane this corresponds to one charge insertion in the boundary and one in the bulk

$$
\left\langle\phi_{-2 q}(0,0) \phi_{q}(x, y)\right\rangle=\left(\frac{2 y}{x^{2}+y^{2}}\right)^{2 \Delta}
$$

Note that in the limit $y \rightarrow 0$ the fusion rules apply and we obtain (3.6) with $\Delta$ replaced by $4 \Delta$.

For the 2-point function in the upper plane we have to consider the 4-point correlation function in the full plane with $q_{1}=q_{2}=-q_{3}=-q_{4}=q$, where $q_{2}$ is inserted symmetrically to $q_{1}$ in relation to the real axis and $q_{4}$ to $q_{3}$. We obtain the bulk correlator

$$
\left\langle\phi_{q}(0, y) \phi_{-q}\left(x, y^{\prime}\right)\right\rangle=\left(\frac{4 y y^{\prime}}{x^{2}\left(x^{2}+\left(y+y^{\prime}\right)^{2}\right)}\right)^{2 \Delta}
$$

Again in the limit $x \rightarrow \infty$ this correlator vanishes. This corresponds to Neumann boundary conditions. The normal derivative to the boundary of (3.8) vanishes on the boundary $\left.\partial_{y}\langle\phi(0) \phi(x)\rangle\right|_{\partial D_{2}}=0$.

For the case of one compactified free boson the process follows in quite a similar way. The main difference resides in the fact that the right and left spectrum charges 

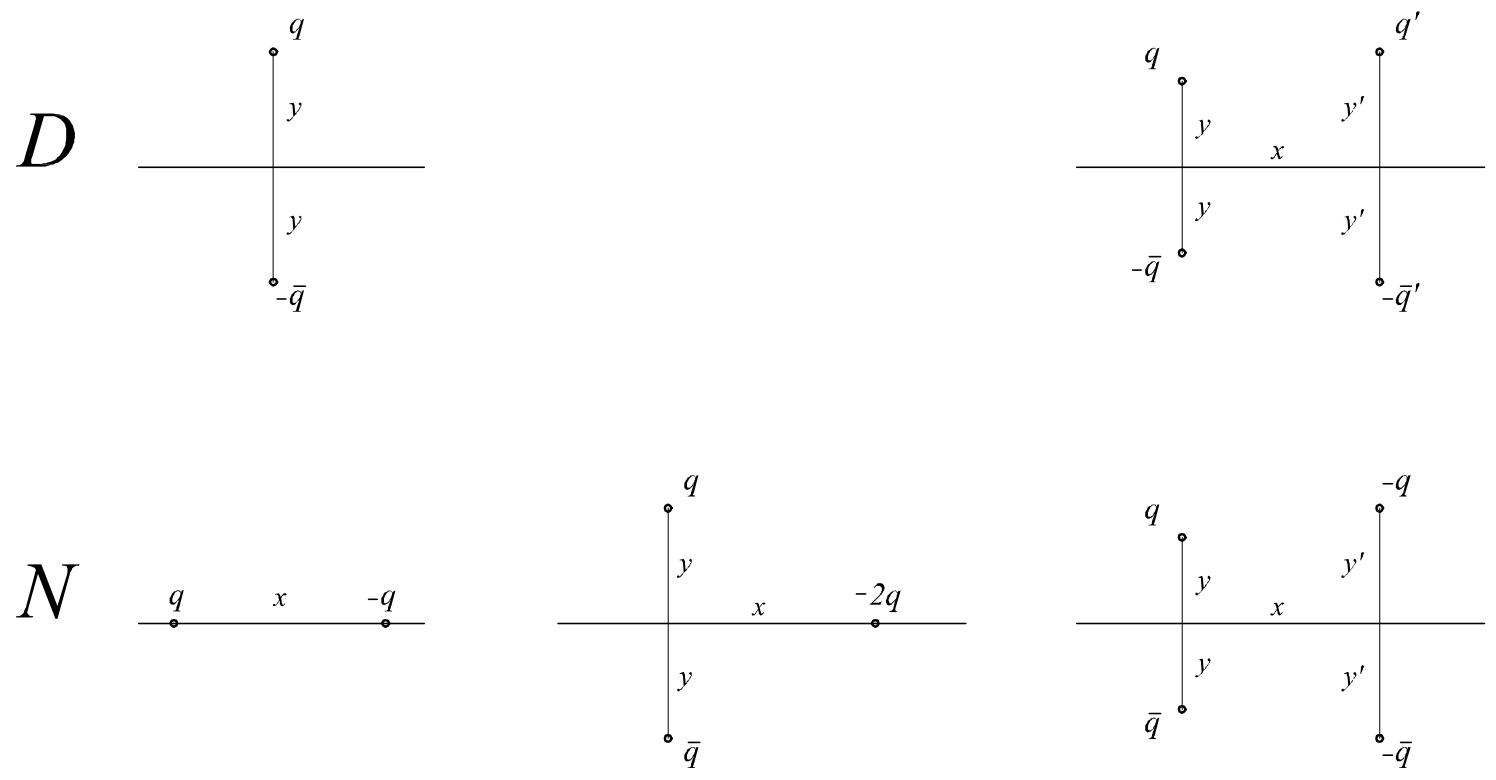

Figure 6: The several possible analytical continuations of the disk (the upper half plane) to the sphere (full plane) holding 2, 3 and 4-point correlation functions for Neumann (N) and Dirichlet (D) boundary conditions.

are different. Taking a charge $q=m+k n / 4$ its image charge is now $\pm \bar{q}$, where $\bar{q}=m-k n / 4$. In this way we have to truncate the spectrum holding $q=-\bar{q}=k n / 4$ for Dirichlet boundary conditions, and $q=\bar{q}=m$ for Neumann boundary conditions, in a pretty similar way as it happens in the Topological Membrane. We summarize in figure 6 the results derived here.

\subsection{Annulus}

We consider the annulus to be a half torus. For simplicity we take the torus to be the region of the complex plane $[-\pi, \pi] \times[0,2 \pi \tau]$ (and the annulus the region $[0, \pi] \times[0,2 \pi \tau])$. We use $z=x+i y$ with $x \in[-\pi, \pi]$ and $y \in[0,2 \pi \tau]$. Here $y$ is the vertical distance (parallel to the imaginary axis) between vertex insertions while $x$ is taken to be the distance to the imaginary axis.

\subsubsection{Dirichlet Boundary Conditions}

Considering mirror charges with opposite sign, 2-point correlations in the torus correspond to the bulk expectation value in the annulus

$$
\langle\phi(x, y)\rangle=\frac{1}{(2 x)^{2 \Delta}}
$$

As in the case of the disk, it blows up in the boundary. But in the boundary this correlation function is not valid since the two charges annihilate each other. Therefore 
the only possible charge insertions in the boundary are $q=0$, that is the identity operator.

Again 3-point correlation functions cannot be used in this case.

For 4-point vertex insertion consider $q_{1}$ and $q_{3}$ inserted to the right of the imaginary axis and $q_{2}$ and $q_{4}$ their mirror charges. The most generic configuration is $q_{1}=-q_{2}=q$ and $q_{3}=-q_{4}=q^{\prime}$ with $z_{1}=-\bar{z}_{2}=x$ and $z_{3}=-\bar{z}_{3}=x^{\prime}+i y$. We obtain the 2-point correlation function in the annulus

$$
\left\langle\phi_{q}(x, 0) \phi_{q^{\prime}}\left(x^{\prime}, y\right)\right\rangle=\frac{1}{(2 x)^{2 \Delta}\left(2 x^{\prime}\right)^{2 \Delta^{\prime}}}\left(1-\frac{4 x x^{\prime}}{\left(x+x^{\prime}\right)^{2}+(y)^{2}}\right)^{2 q q^{\prime} / k}
$$

Again the same arguments used for the disk apply. There cannot exist boundary insertions other than the identity and the tangential derivative to the boundary $\left.\partial_{y}\langle\phi\rangle\right|_{\partial C_{2}}=0$ vanish.

\subsubsection{Neumann Boundary Conditions}

Considering now the mirror charges having the same sign, again the fields in the bulk have zero expectation value. But the 2-point boundary correlation function is computed to be

$$
\left\langle\phi_{q}(0,0) \phi_{-q}(\pi, y)\right\rangle=\frac{1}{\pi^{2 \Delta}+y^{2 \Delta}}
$$

where we take one insertion in each boundary. In the case that the insertions are in the same boundary the factor of $\pi^{2 \Delta}$ is absent.

The 3-point function in the torus corresponds either to 2-point function in the annulus (taking only one insertion in the boundary) or to 3-point function (taking all the insertions in the boundaries). Taking one insertion in the bulk $\phi_{q}(x, 0)$ (with mirror image $\left.\phi_{q}(-x, 0)\right)$ and other in the boundary $\phi_{-2 q}(\pi, y)$ we obtain

$$
\left\langle\phi_{q}(x, 0) \phi_{-2 q}(\pi, y)\right\rangle=\left(\frac{x}{\pi^{2}+x^{2}+y^{2}}\right)^{2 \Delta}
$$

If the insertion is the boundary $x=0$ the factor of $\pi^{2}$ is absent.

The 4-point function in the torus corresponds in the annulus either to a2-point function (bulk insertions), 3-point function (two vertices in the boundaries) or 4point function (all vertices in the boundary). Taking all vertex insertions in the bulk as pictured in figure 7 we obtain

$$
\left\langle\phi_{q}(x, 0) \phi_{-q}\left(x^{\prime}, y\right)\right\rangle=\left(\frac{4 x x^{\prime}}{\left(\left(x-x^{\prime}\right)^{2}+y^{2}\right)\left(\left(x+x^{\prime}\right)^{2}+y^{2}\right)}\right)^{2 \Delta}
$$

As an example of two insertions in the boundaries take them to be both in the boundary $x=0$, we obtain

$$
\left\langle\phi_{q}(x, 0) \phi_{q^{\prime}}(0, y) \phi_{-q-q^{\prime}}\left(0, y+y^{\prime}\right)\right\rangle=\frac{1}{y^{2 \Delta^{\prime}}}\left(\frac{2 x}{\left(x^{2}+y^{\prime 2}\right)^{2}}\right)^{2 \Delta}\left(\frac{x^{2}+\left(y+y^{\prime}\right)^{2}}{y^{2}\left(x^{2}+y^{\prime 2}\right)}\right)^{2 q q^{\prime} / k}
$$



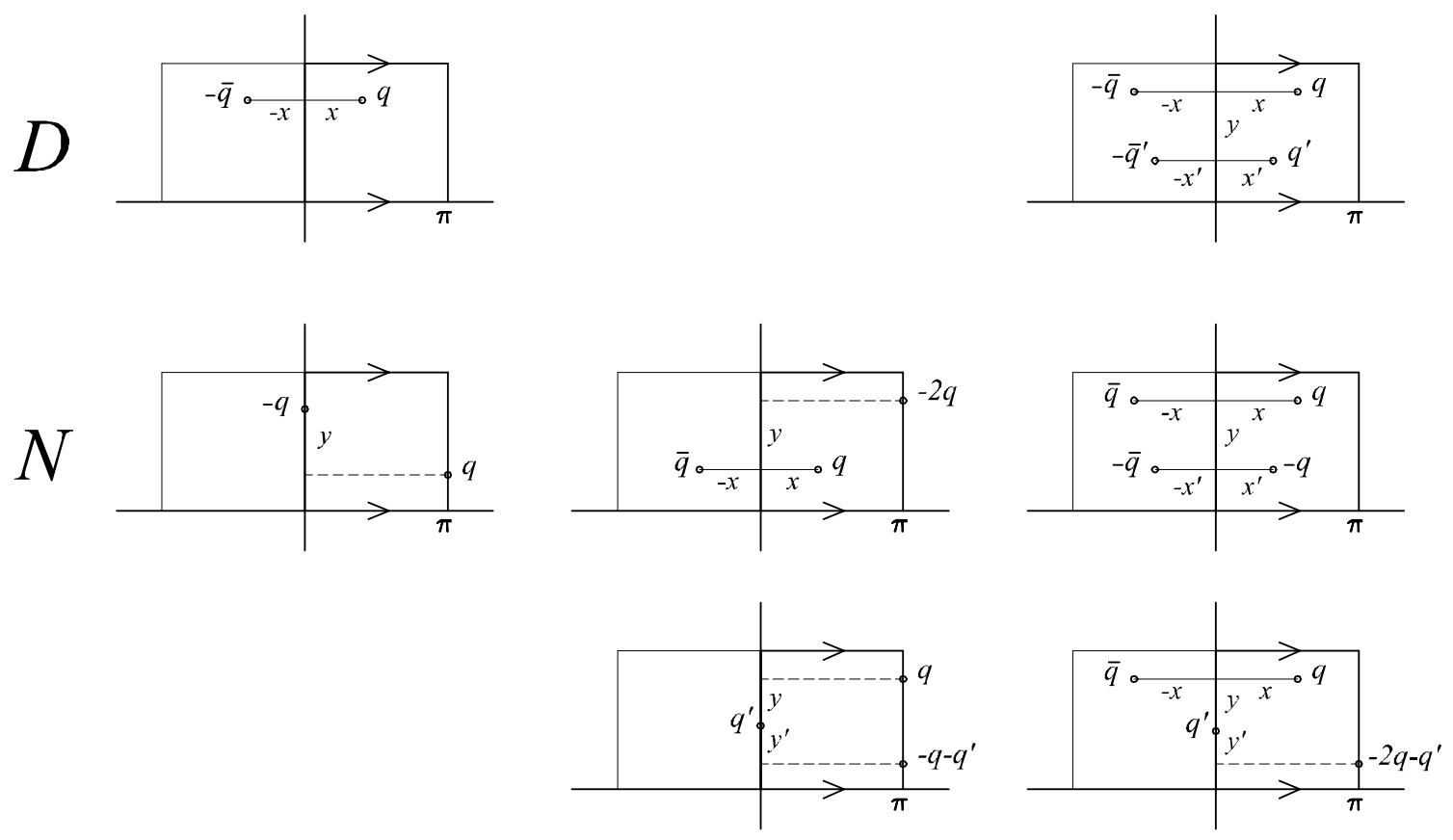

Figure 7: The several possible analytical continuations of the annulus to the torus holding 2, 3 and 4-point correlation functions, for Neumann (N) and Dirichlet (D) boundary conditions.

We can stop here, for our purposes it is not necessary to exhaustively enumerate all the possible cases. As expected the normal derivative to the boundary of these correlation functions $\left(\partial_{x}\langle\ldots\rangle\right)$ vanishes at the boundary. These results are summarized in figure 7 .

For the case of one compactified free boson the process follows as explained before. The spectrum must be truncated holding $q=-\bar{q}=k n / 4$ for Dirichlet boundary conditions and $q=\bar{q}=m$ for Neumann boundary conditions.

\section{4. $\operatorname{TM}(\mathrm{GT})$}

Is now time to turn to the $3 D \mathrm{TM}(\mathrm{GT})$. In this section we present results derived directly from the bulk theory and its properties. The derivations of the results presented here are in agreement with the CFT arguments in the last section.

Take for the moment a single compact $U(1)$ TMGT corresponding to $c=1 \mathrm{CFT}$ with action

$$
S=\int_{M} d t d^{2} z\left[-\frac{\sqrt{-g}}{\gamma} F_{\mu \nu} F^{\mu \nu}+\frac{k}{8 \pi} \epsilon^{\mu \nu \lambda} A_{\mu} F_{\nu \lambda}\right]
$$

where $M=\Sigma \times[0,1]$ has two boundaries $\Sigma_{0}$ and $\Sigma_{1} . \Sigma$ is taken to be a compact manifold, $t$ is in the interval $[0,1]$ and $(z, \bar{z})$ stand for complex coordinates on $\Sigma$. From now on we will use them by default. 
As widely known this theory induces new degrees of freedom in the boundaries, which are fields belonging to $2 D$ chiral CFT's theories living on $\Sigma_{0}$ and $\Sigma_{1}$.

The electric and magnetic fields are defined as

$$
\begin{aligned}
E^{i} & =\frac{1}{\gamma} F^{0 i} \\
B & =\partial_{z} A_{\bar{z}}-\partial_{\bar{z}} A_{z}
\end{aligned}
$$

and the Gauss law is simply

$$
\partial_{i} E^{i}+\frac{k}{4 \pi} B=\rho_{0}
$$

Upon quantization the charge spectrum is

$$
Q=m+\frac{k}{4} n
$$

for some integers $m$ and $n$. Furthermore it has been proven in $[32,39]$ that, for compact gauge groups and under the correct relative boundary conditions, one insertion of $Q$ on one boundary (corresponding to a vertex operator insertion on the boundary CFT) will, necessarily, demand an insertion of the charge

$$
\bar{Q}=m-\frac{k}{4} n
$$

on the other boundary. We are assuming this fact through the rest of this paper.

Our aim is to orbifold TM theory in a similar way to Horava [47], who obtained open boundary world-sheets through this construction. We are going to take a path integral approach and reinterpret it in terms of discrete $P T$ and $P C T$ symmetries of the bulk $3 D \mathrm{TM}(\mathrm{GT})$.

\subsection{Horava Approach to Open World-Sheets}

Obtaining open string theories out of $3 D$ (topological) gauge theories means building a theory in a manifold which has boundaries (the $2 D$ open string world-sheet) that is already a boundary (of the $3 D$ manifold). This construction raises a problem since the boundary of a boundary is necessarily a null space. One interesting way out of this dilemma is to orbifold the $3 D$ theory, then its singular points work as the boundary of the $2 D$ boundary. Horava [47] introduced an orbifold group $G$ that combines the world-sheet parity symmetry group $Z_{2}^{W S}(2 D)$ with two elements $\{1, \Omega\}$, together with a target symmetry $\tilde{G}$ of the $3 D$ theory fields

$$
G \subset \tilde{G} \times Z_{2}^{W S}
$$

With this construction we can get three different kind of constructions. Elements of the kind $h=\tilde{h} \times 1_{Z_{2}^{W S}}$ induce twists in the target space (not acting in the world-sheet at all), for elements $\omega=1_{\tilde{G}} \times \Omega$ we orbifold the world-sheet manifold (getting an 
open world-sheet) without touching in the target space and for elements $g_{1}=\tilde{g}_{1} \times \Omega$ we obtain exotic world-sheet orbifold. In this last case it is further necessary to have an element corresponding to the twist in the opposite direction $g_{2}=\tilde{g_{2}} \times \Omega$. To specify these twists on some world-sheet it is necessary to define the monodromies of fields on it. Taking the open string $C_{o}=C / Z_{2}$ as the orbifold of the closed string $C$

$$
\pi\left(C_{o}\right)=D \equiv Z_{2} * Z_{2} \equiv Z_{2} \otimes Z
$$

* being the free product and $\varangle$ the semidirect product of groups. $D$ is the infinite dihedral group, the open string first homotopy group. So the monodromies of fields in $C_{o}$ corresponds to a representation of this group in the orbifold group, $Z_{2} * Z_{2} \rightarrow G$, such that the commutative triangle

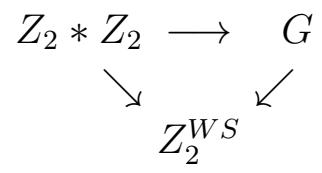

is complete. The partition function contains the sum over all possible monodromies

$$
Z_{C}(\tau)=\frac{1}{|G|} \sum_{g_{1}, g_{2}, h} Z_{C}\left(g_{1}, g_{2}, h ; \tau\right)
$$

where $\tau$ is the moduli of the manifold. The monodromies $g_{1}, g_{2}$ and $h$ are elements of $G$ as previously defined satisfying $g_{i}^{2}=1$ and $\left[g_{i}, h\right]=1$.

It will be shown that PCT plays the role of one of such symmetries with $g_{1}=g_{2}$. It is in this sense one of the most simple cases of exotic world-sheet orbifolds.

The string amplitudes can be computed in two different pictures. The loopchannel corresponds to loops with length $\tau$ of closed and open strings and the amplitudes are computed as traces over the Hilbert space. The tree-channel corresponds to a cylinder of length $\tilde{\tau}$ created from and annihilated to the vacua through boundary $(|B\rangle)$ and/or crosscaps $(|C\rangle)$ states. Comparing both ways for the same amplitudes we obtain

$$
\begin{aligned}
& \text { Annulus }\left(C_{2}\right): \operatorname{Tr}_{\text {open }}\left(e^{-H_{o} \tau}\right)=\left\langle B\left|e^{-H_{c} \tilde{\tau}}\right| B\right\rangle \\
& \text { Möbius Strip }\left(M_{2}\right): \operatorname{Tr}_{\text {open }}\left(\Omega e^{-H_{o} \tau}\right)=\frac{1}{2}\left\langle B\left|e^{-H_{c} \tilde{\tau}}\right| C\right\rangle+\frac{1}{2}\left\langle C\left|e^{-H_{c} \tilde{\tau}}\right| B\right\rangle \\
& \text { Klein Bottle }\left(K_{2}\right): \operatorname{Tr}_{\text {open }}\left(\Omega e^{-H_{o} \tau}\right)=\left\langle C\left|e^{-H_{c} \tilde{\tau}}\right| C\right\rangle
\end{aligned}
$$

These equations constitute constraints similar to the modular invariance constraints of closed string theories. The relation between the moduli are, for $K_{2}$ and $M_{2}$ $\tau=1 /(2 \tilde{\tau})$, and for $C_{2} \tau=2 /(\tilde{\tau})$.

In terms of manifolds it is intended to obtain some open boundary $\Sigma_{o}=\Sigma / I$ (where boundary refers to $M=\Sigma \times[0,1]$ ) which is the involution under the symmetry $I$ of its double, $\Sigma$. The resulting orbifolded manifold is

$$
M_{o}=(\Sigma \times[0,1]) / I
$$




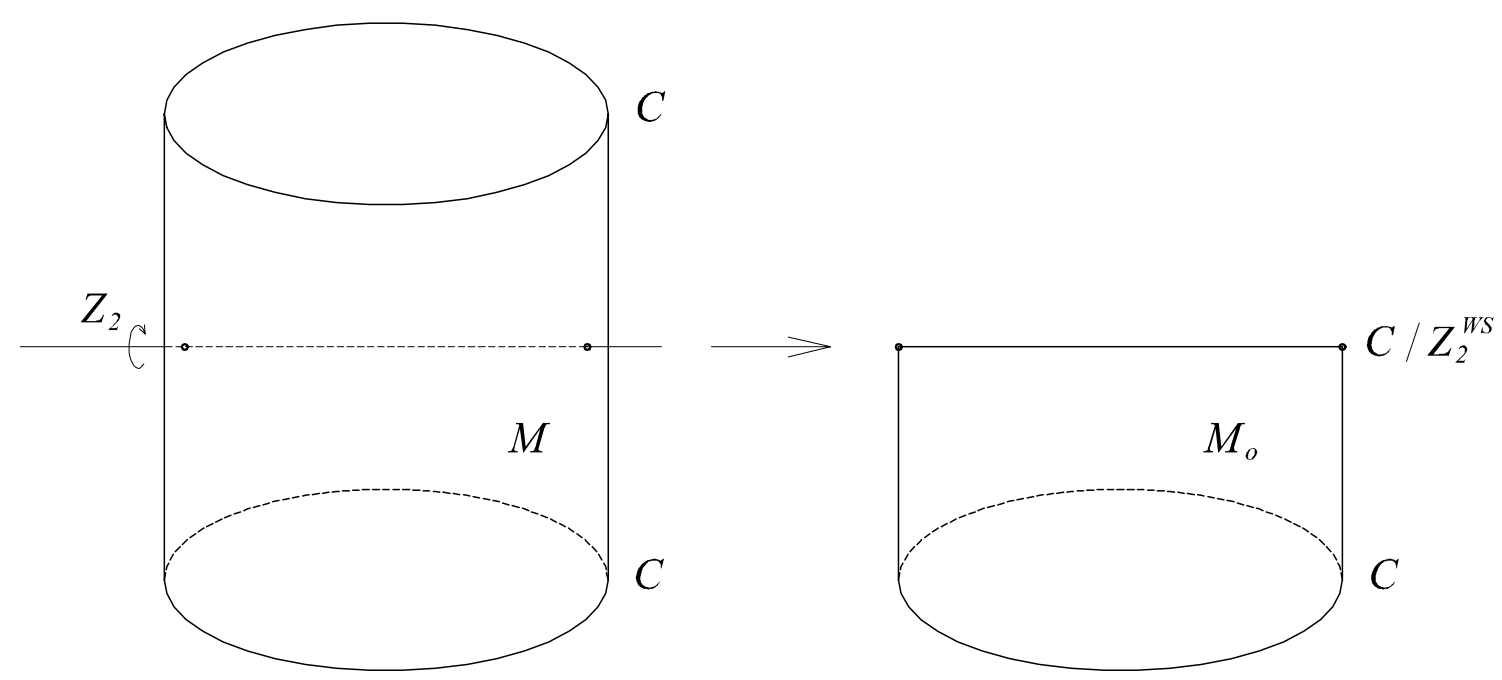

Figure 8: The thickened open string $C_{o}$ as an orbifold of the thickened closed string $C$ under a $I=Z_{2}$ symmetry. The boundaries of $C_{o}=C / Z_{2}^{W S}$ are the singular points of the orbifold.

where $I$ acts in $t$ as Time Inversion $t \rightarrow 1-t$. This construction is presented in figure 8 .

In terms of the action and fields in the theory Horava used the same approach of extending them to the doubled manifold

$$
2 S_{o}\left(A_{o}\right)=S(A)
$$

In simple terms $A$ stands for the extension of $A_{o}$ from $M_{o}$ to its double $M$.

Since there is a one-to-one correspondence between the quantum states of the gauge theory on $\mathrm{M}$ and the blocks of the WZNW model, we may write

$$
Z_{\Sigma}=\sum h_{i j} \Psi_{i} \otimes \bar{\Psi}_{j} \in \mathcal{H}_{\Sigma} \otimes \overline{\mathcal{H}}_{\Sigma}
$$

where $\Psi_{i}$ stands for a basis of the Hilbert space $\mathcal{H}_{\Sigma}$. The open string counterpart in the orbifolded theory is

$$
Z_{\Sigma_{o}}=\sum a_{i} \Psi_{i} \in \mathcal{H}_{\Sigma}
$$

which also agrees with the fact that in open CFT's the partition function is the sum of characters (instead of the sum of squares) due to the holomorphic and antiholomorphic sectors not being independent.

\subsection{Discrete Symmetries and Orbifold of TM(GT)}

Following the discussion of section 2 and section 1.1, it becomes obvious that the parity operation plays a fundamental role in obtaining open and/or non-orientable manifolds out of closed orientable ones. Hence obtaining open/unorientable theories out of closed orientable theories. 
Generally there are several ways of defining parity. The ones we are interested in have already been presented here. For the usual ones, $P_{1}$ and $\Omega$ defined in (2.4) and (2.15), the fields of our $3 D$ theory transform like

$$
\begin{array}{rlrl}
P_{1}: z & \leftrightarrow \bar{z} & \Omega: z & \leftrightarrow-\bar{z} \\
\Lambda & \rightarrow \Lambda & \Lambda & \rightarrow \Lambda \\
A_{0} & \rightarrow A_{0} & A_{0} \rightarrow A_{0} \\
A_{z} & \leftrightarrow A_{\bar{z}} & A_{z} & \leftrightarrow-A_{\bar{z}} \\
E^{z} & \leftrightarrow E^{\bar{z}} & & E^{z} \leftrightarrow-E^{\bar{z}} \\
B & \rightarrow-B & B & \rightarrow-B \\
Q & \rightarrow Q & Q & \rightarrow Q
\end{array}
$$

where $\Lambda$ is the gauge parameter entering into $U(1)$ gauge transformations. Under these two transformations the action transforms as

$$
\int\left(F^{2}+k A \wedge F\right) \rightarrow \int\left(F^{2}-k A \wedge F\right)
$$

The theory is clearly not parity invariant. Let us then look for further discrete symmetries which we may combine with parity in order to make the action (theory) invariant. Introduce time-inversion, $T: t \rightarrow 1-t$, implemented in this non-standard way due to the compactness of time. Note that $t=1 / 2$ is a fixed point of this operation. Upon identification of the boundaries as described in [39] the boundary becomes a fixed point as well. It remains to define how the fields of the theory change under this symmetry. There are two possible transformations compatible with gauge transformations, $A_{\Lambda}(t, z, \bar{z})=A(t, z, \bar{z})+\partial \Lambda(t, z, \bar{z})$. They are:

$$
\begin{aligned}
C T: & \rightarrow 1-t \\
\Lambda & \rightarrow \Lambda \\
A_{0} & \rightarrow-A_{0} \\
\mathbf{A} & \rightarrow \mathbf{A} \\
\mathbf{E} & \rightarrow-\mathbf{E} \\
B & \rightarrow B \\
Q & \rightarrow
\end{aligned}
$$

and

$$
\begin{aligned}
T: & \rightarrow 1-t \\
\Lambda & \rightarrow-\Lambda \\
A_{0} & \rightarrow A_{0} \\
\mathbf{A} & \rightarrow-\mathbf{A} \\
\mathbf{E} & \rightarrow \mathbf{E} \\
B & \rightarrow-B \\
Q & \rightarrow-Q
\end{aligned}
$$



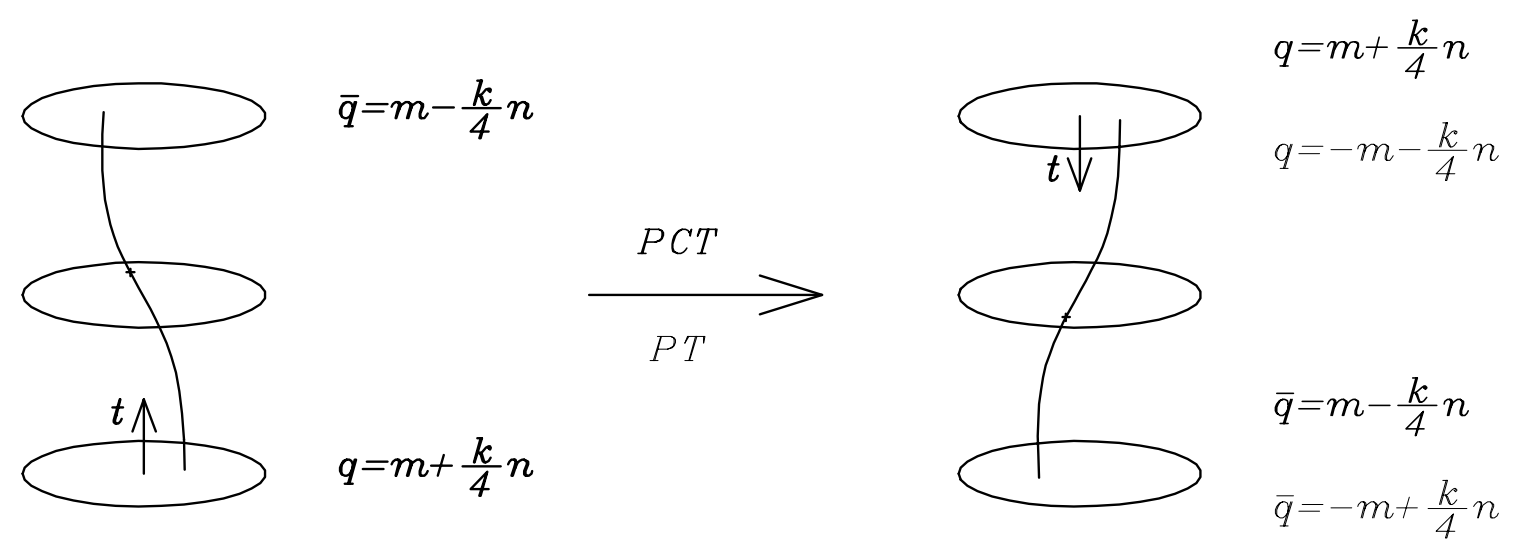

Figure 9: Exchange of boundaries due to $P T / P C T$ transformation.

where we defined $C$, charge conjugation, as $A_{\mu} \rightarrow-A_{\mu}$. This symmetry inverts the sign of the charge, $Q \rightarrow-Q$, as usual. These discrete symmetries together with parity $P$ or $\Omega$ are the common ones used in $3 D$ Quantum Field Theory. When referring to parity in generic terms we will use the letter $P$.

Under any of the $T$ and $C T$ symmetries the action changes in the same fashion it does for parity $P$, as given by (4.16). In this way any of the combinations $P T$ and $P C T$ are symmetries of the action, $S \rightarrow S$. Gauging them is a promising approach to define the $\mathrm{TM}(\mathrm{GT})$ orbifolding. It is now clear why we need extra symmetries, besides parity, in order to have combinations of them under which the theory (action) is invariant. In general, whatever parity definition we use, these results imply that $P T$ and $P C T$ are indeed symmetries of the theory.

We can conclude straight away that any of the two previous symmetries exchange physically two boundaries working as a mirror transformation with fixed point $(t=1 / 2, z=\bar{z}=x)$ (corresponds actually to a line) as pictured in figure 9 . We are considering that, whenever there is a charge insertion in one boundary of $\mathrm{q}=\mathrm{m}+\mathrm{kn} / 4$, it will exist an insertion of $\bar{q}=m-k n / 4$ in the other boundary [30,39].

Under the symmetries PT and PCT as given by (4.15), (4.17) and (4.18) the boundaries will be exchanged as presented in figure 9. In the case of $P C T$ the charges will simply be swaped but in the case of $P T$ their sign will be change $q \rightarrow-q$. Note that $\Sigma_{\frac{1}{2}}=\Sigma(t=1 / 2)$ only feels $P$ or $C P$.

As will be shown in detail there are important differences between the two symmetries $C T$ and $T$, they will effectively gauge field configurations corresponding to untwisted/twisted sectors of closed strings and Neumann/Dirichlet boundary conditions of open strings.

Not forgetting that our final aim is to orbifold/quotient our theory by gauging the discrete symmetries, let us proceed to check compatibility with the desired symmetries in detail. It is important to stress that field configurations satisfying any 

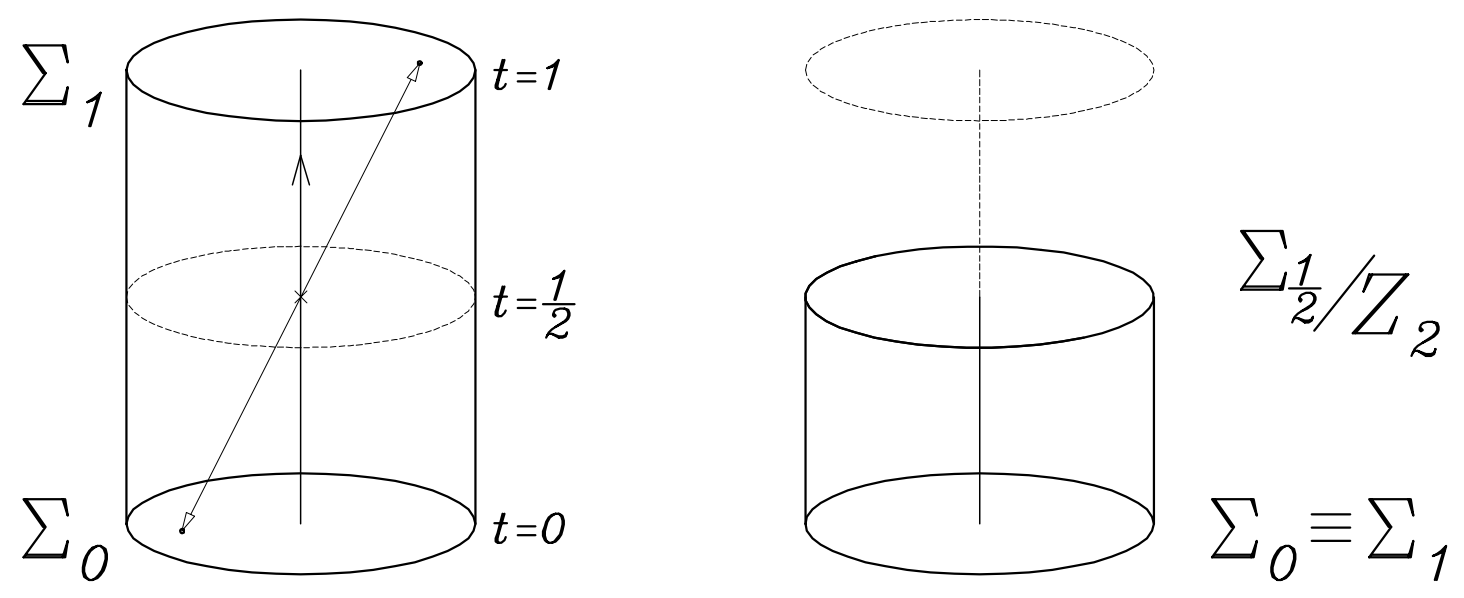

Figure 10: Orbifolding of $\mathrm{TM}(\mathrm{GT}) . \Sigma_{\frac{1}{2}}$ only feels $P T$ or $P$ which are isomorphic to $Z_{2}$

PT / PCT combinations of the previous symmetries exist, in principle, from the start in the theory. We can either impose by hand that the physical fields obey one of them (as is usual in QFT) or we can assume that we have a wide theory with all of these field configurations and obtain (self consistent) subtheories by building suitable projection operators that select some type of configurations. It is precisely this last construction that we have in mind when building several different theories out of one. In other words we are going to build different new theories by gauging discrete symmetries of the type PCT and PT.

It is important to stress what the orbifold means in terms of the boundaries and bulk from the point of view of $\mathrm{TM}(\mathrm{GT})$. It is splitting the manifold $M$ into two pieces creating one new boundary at $t=1 / 2$. This boundary is going to feel only $C P$ or $P$ symmetries since it is located at the temporal fixed point of the orbifold. Figure 10 shows this procedure. In this way this new boundary is going to constrain the new theory in such a way that the boundary theories will correspond to open and unoriented versions of the original full theory.

\subsection{Tree Level Amplitudes for Open and Closed Unoriented Strings}

We start by considering tree level approximation to string amplitudes, i.e. the Riemann surfaces are of genus 0 . These surfaces are the sphere (closed oriented strings) and its orbifolds: the disk (open oriented) and the projective plane (closed unoriented) as was discussed in section 2. From the point of view of $\operatorname{TM}(\mathrm{GT})$, orbifolding means that we split the manifold $M$ into two pieces that are identified. As a result at $t=1 / 2$, the fixed point of the orbifold, a new boundary is created.

For different orbifolds we shall have different admissible field configurations. In the following discussion we studied which are the configurations compatible with $P T$ 
and $P C T$ for the several parity operations already introduced.

\subsubsection{Disk}

Let us start from the simplest case - the disk is obtained by the involution of the sphere under $P_{1}$ as given by (2.4). So consider the identifications under $P_{1} C T$ and $P_{1} T$. For the first one the fields relate as

$$
\begin{aligned}
P_{1} C T: \quad(t, z, \bar{z}) & =\Lambda(1-t, \bar{z}, z) \\
\partial_{i} E^{i}(t, z, \bar{z}) & =-\partial_{i} E^{i}(1-t, \bar{z}, z) \\
B(t, z, \bar{z}) & =-B(1-t, \bar{z}, z) \\
Q(t)=\int_{\Sigma(t)} \rho_{0} & =Q(1-t)=-\int_{\tilde{\Sigma}(1-t)}\left(-\rho_{0}\right)
\end{aligned}
$$

The orientations of $\Sigma$ and $\tilde{\Sigma}$ are opposite. Under these relations the Wilson lines have the property

$$
\exp \left\{i Q \int_{C} d x^{\mu} A_{\nu}\right\}=\exp \left\{i Q \int_{-C} d x^{\mu} A_{\nu}\right\}
$$

This means that for the configurations obeying the relations (4.19) we loose the notion of time direction.

Under the involution of our $3 D$ manifold, using the above relations as geometrical identifications, the boundary becomes $t=0$ and $t=1 / 2$. For the moment let us check the compatibility of the observables with the proposed orbifold constructions given by the previous relations. In a very naive and straightforward way, when we use $P_{1} C T$ as given by (4.19) the charges should maintain their sign $(q(t) \cong q(1-t))$. Then by exchanging boundaries we need to truncate the spectrum and set $q \cong \bar{q}=m$ in order the identification to make sense. Let us check what happens at the singular point of our orbifolded theory, $t=1 / 2$. The fields are identified according to the previous rules but the manifold $\Sigma(t=1 / 2)=S^{2}$ is only affected by $P_{1}$.

Take two Wilson lines that pierce the manifold in two distinct points, $z$ and $z^{\prime}$. Under the previous involution $P_{1} C T, z$ is identified with $\bar{z}$ for $t=1 / 2$. Then, geometrically, we must have $z^{\prime}=\bar{z}$ in order to have spatial identification of the piercings. The problem is that when we have only two Wilson lines, TM(GT) demands that they carry opposite charges. In order to implement the desired identification we are left with $q=0$ as the only possibility. For the case where the Wilson lines pierce the manifold in the real axis, $z=x$ and $z^{\prime}=x^{\prime}$, the involution is possible as pictured in figure 11 since we identify $x \cong x$ and $x^{\prime} \cong x^{\prime}$.

In the presence of three Wilson lines, following the same line of arguing, we will necessarily have one insertion in the boundary and two in the bulk as pictured in figure 12. Only in the presence of four Wilson lines, as pictured in figure 13 can we avoid any insertion in the boundary. 


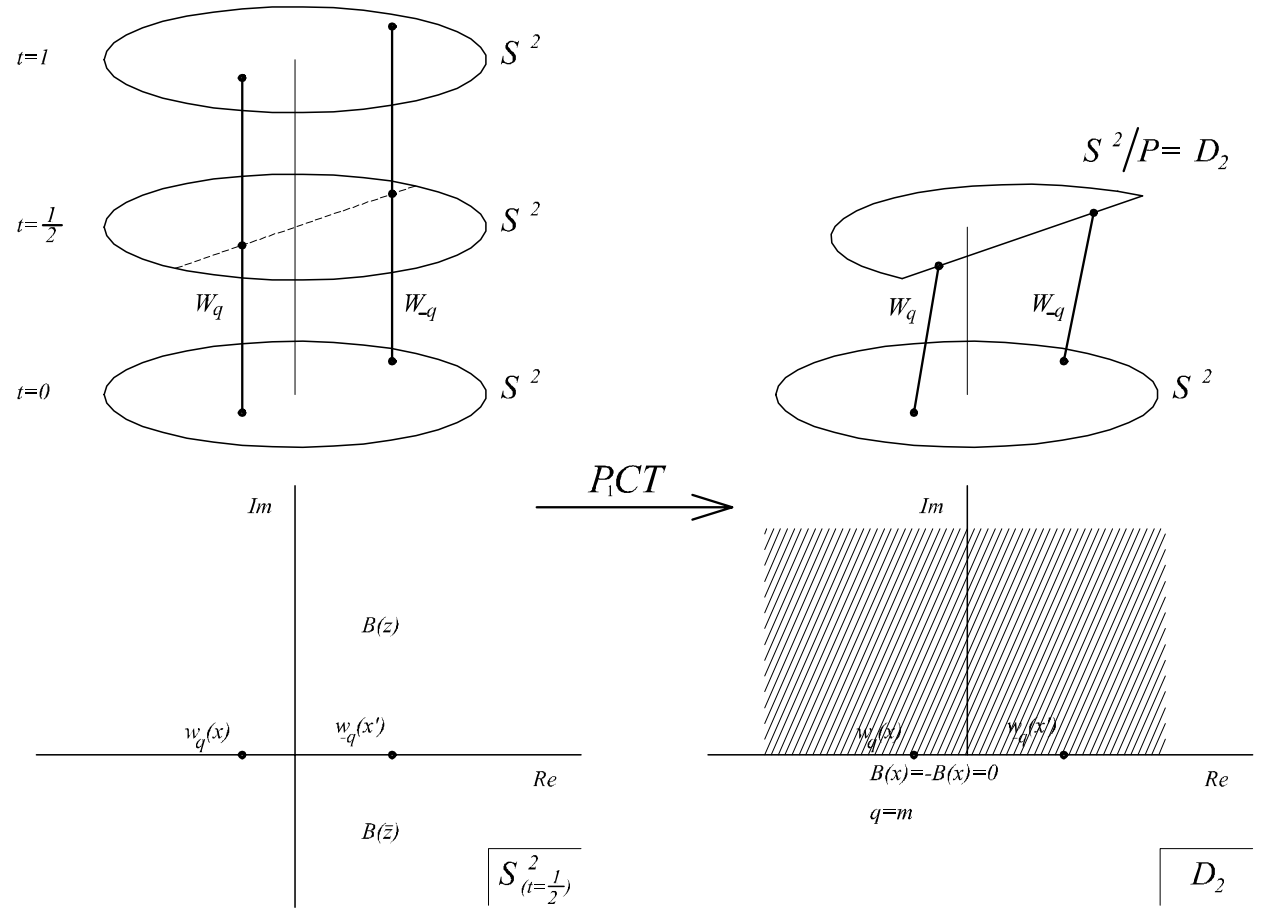

Figure 11: Orbifold under $P_{1} C T$ in the presence of 2 Wilson lines, $W_{q}$ and $W_{-q}$. They need to pierce $\Sigma_{o \frac{1}{2}}=S^{2} / P_{1}=D_{2}$ in the real axis and the allowed charges are $q=m$.

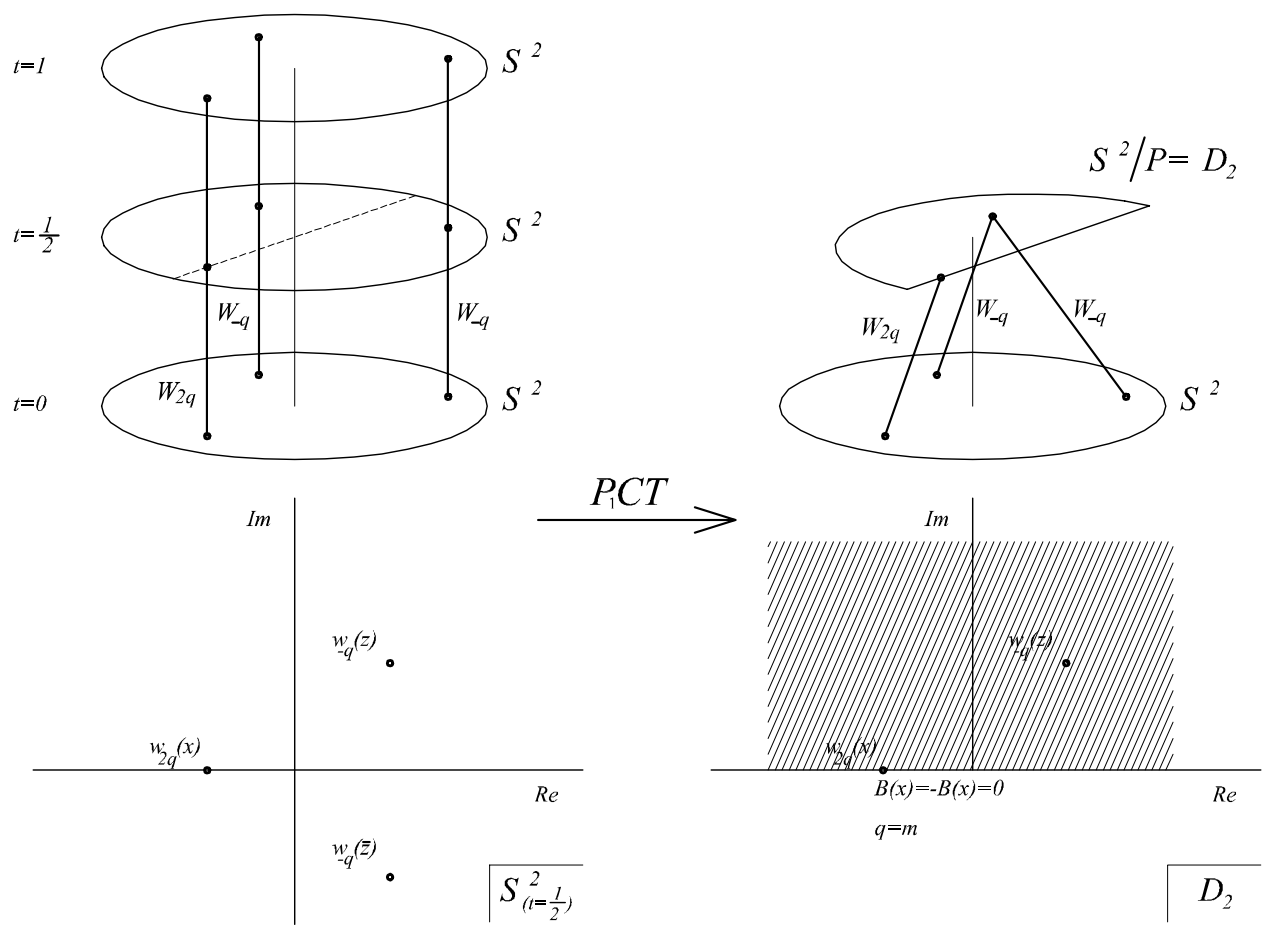

Figure 12: Orbifold under $P_{1} C T$ in the presence of 3 Wilson lines, $W_{2 q}$ and two $W_{-q}$. $W_{2 q}$ must pierce $\Sigma_{o \frac{1}{2}}=S^{2} / P_{1}=D_{2}$ in the real axis and the allowed charges are $q=m$. 


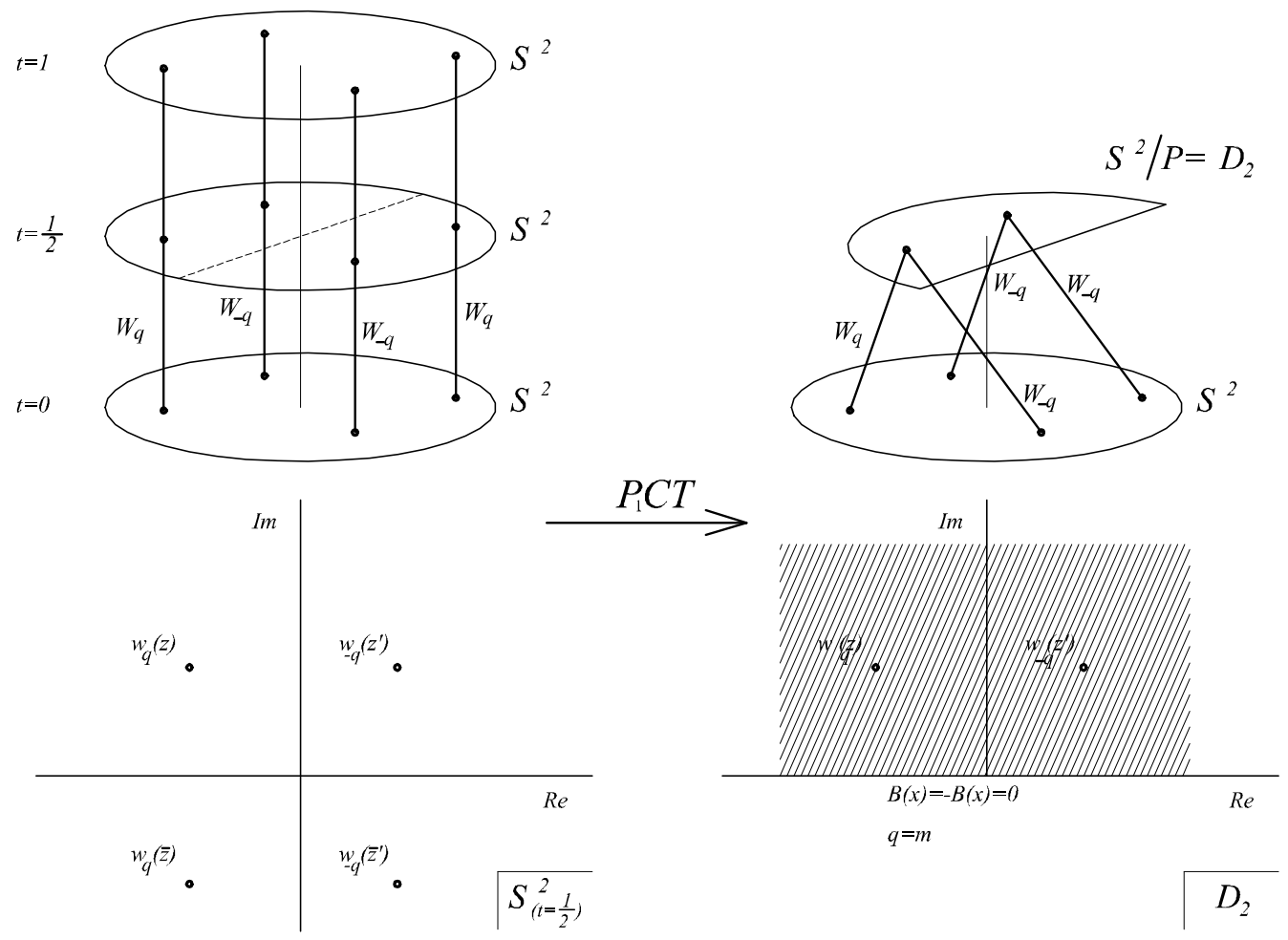

Figure 13: Orbifold under $P_{1} C T$ in the presence of 4 Wilson lines, two $W_{q}$ and two $W_{-q}$. The allowed charges are $q=m$.

Note that the identification $B(z, \bar{z}) \cong-B(\bar{z}, z)$ in the real axis implies necessarily $B(x, x)=0$. Remember that $2 \pi n=\int B$ (see [32,39] for details). We could as well have an insertion in the boundary and one in the bulk

This fact is simply the statement that by imposing $P_{1} C T$ we are actually imposing Neumann boundary conditions. The charges of the theory become $q=m$, this means that the string spectrum has only Kaluza-Klein momenta. Furthermore the monopole induced processes are suppressed, recall that they change the charge by an amount $k n / 2$ which would take the charges out of the spectrum allowed in this configurations.

Following our journey consider next $P_{1} T$. The fields now are related in the following way

$$
\begin{aligned}
P_{1} T: \Lambda(t, z, \bar{z}) & =-\Lambda(1-t, \bar{z}, z) \\
\partial_{i} E^{i}(t, z, \bar{z}) & =\partial_{i} E^{i}(1-t, \bar{z}, z) \\
B(t, z, \bar{z}) & =B(1-t, \bar{z}, z) \\
Q=\int_{\Sigma(t)} \rho_{0} & =-Q=-\int_{\tilde{\Sigma}(1-t)}\left(\rho_{0}\right)
\end{aligned}
$$

The Wilson line has the same property $(4.20)$ as in the previous case.

Now the charges change sign under a $P_{1} T$ symmetry. As before identifying the 
charges in opposite boundaries truncates the spectrum, $q(t) \cong-q(1-t)$. So we must have $q \cong-\bar{q}=n k / 4$.

We can, in this case identify two piercings in the bulk since the charge identifications are now $q \cong-q$ is compatible with $\mathrm{TM}(\mathrm{GT})$. But we cannot insert any operator other than the identity $\phi_{0}$ in the real axis since the corresponding charge must be zero $q(x)=-q(x)=0$. Therefore this kind of orbifolding is only possible when we have a even number of Wilson lines propagating in the bulk. The result for two Wilson lines is pictured in figure 14 and for four in figure 15 .

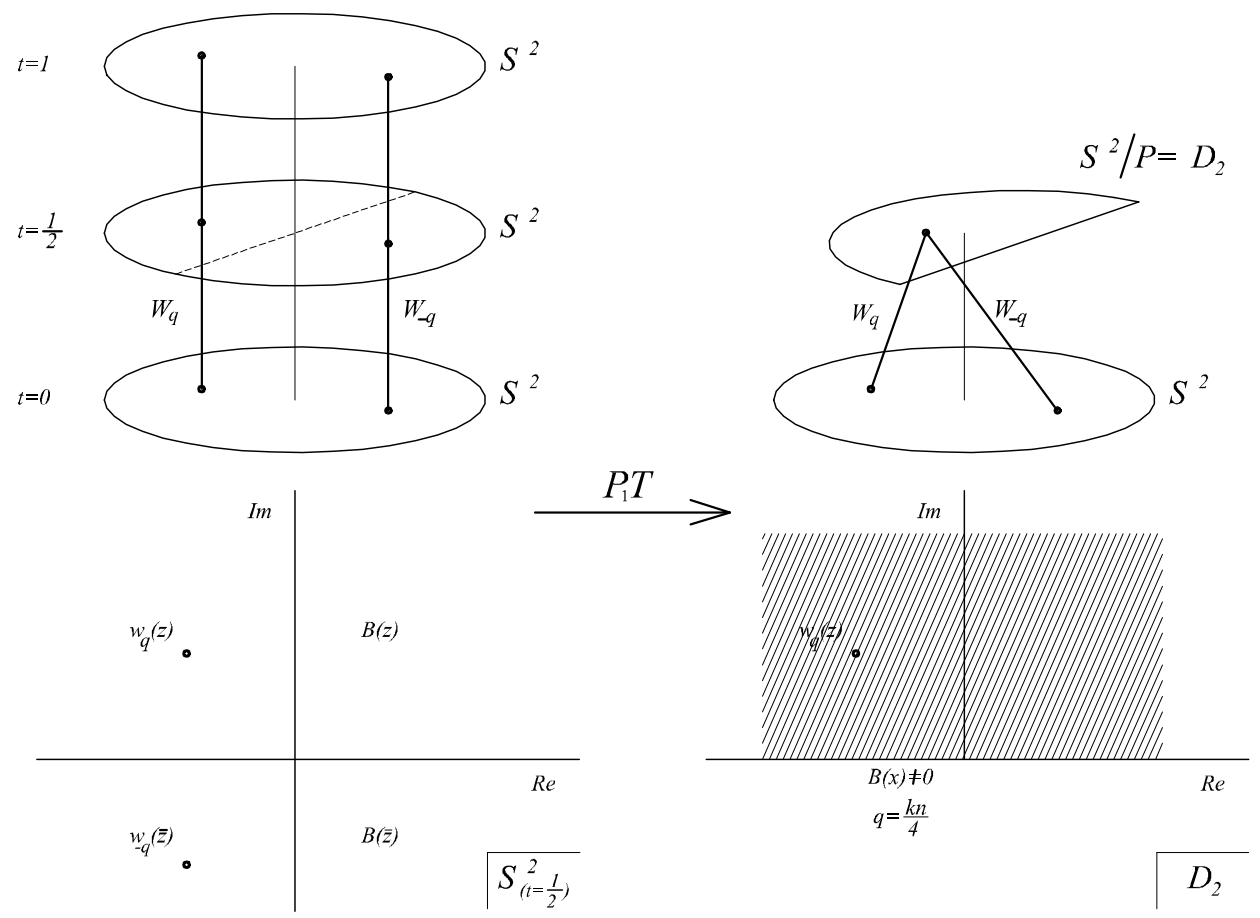

Figure 14: Orbifold under $P_{1} T$ in the presence of 2 Wilson lines, $W_{q}$ and $W_{-q}$. The new boundary is $\Sigma_{o \frac{1}{2}}=S^{2} / P_{1}=D_{2}$. The allowed charges are $q=k n / 4$.

In terms of the full theory, we just define a new $2 D$ boundary which is a disk. The piercings of Wilson lines are none others than vertex operators (or fields) of a Conformal Field Theory defined on the Disk. In this case $B(z, \bar{z})=B(\bar{z}, z)$, then $B \neq 0$ in the boundary.

So this facts translates into Dirichlet boundary conditions for $P_{1} T$. The charges allowed are $q=n k / 4$, the winding number of string theory. The monopole induced processes are now allowed being crucial in this construction since they allow the gluing in this new boundary of two Wilson lines carrying charges $q=n k / 4$ and $\bar{q}=-n k / 4$. 


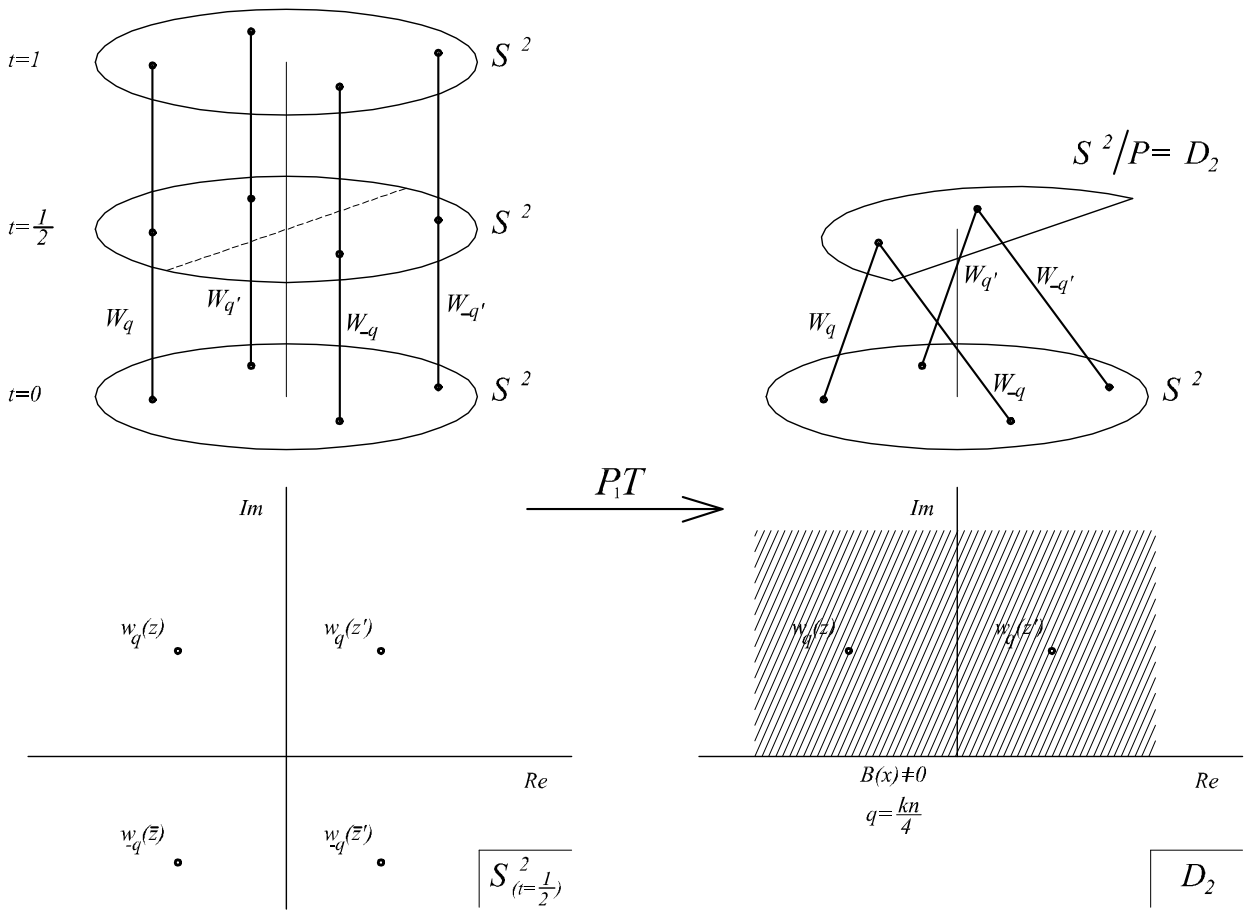

Figure 15: Orbifold under $P_{1} T$ in the presence of 4 Wilson lines, $W_{q}, W_{q}^{\prime}, W_{-q}$ and $W_{-q^{\prime}}$. The new boundary is $\Sigma_{o \frac{1}{2}}=S^{2} / P_{1}=D_{2}$. The allowed charges are $q=k n / 4$.

\subsubsection{Projective Plane}

We now consider the parity operation as the antipodal identification given in (2.8). We thus obtain the projective plane as the new $2 d$ boundary of $\mathrm{TM}(\mathrm{GT})$.

The transformation is given by

$$
\begin{aligned}
P_{2}: \quad z & \rightarrow-\frac{1}{\bar{z}} \\
\bar{z} & \rightarrow-\frac{1}{\bar{z}} \\
\Lambda(z, \bar{z}) & \rightarrow \Lambda\left(-\frac{1}{\bar{z}},-\frac{1}{\bar{z}}\right) \\
A_{z}(z, \bar{z}) & \rightarrow \frac{1}{z^{2}} A_{\bar{z}}\left(-\frac{1}{\bar{z}},-\frac{1}{z}\right) \\
A_{\bar{z}}(z, \bar{z}) & \rightarrow \frac{1}{\bar{z}^{2}} A_{z}\left(-\frac{1}{\bar{z}},-\frac{1}{z}\right) \\
B(z, \bar{z}) & \rightarrow \frac{1}{z^{2} \bar{z}^{2}} B\left(-\frac{1}{\bar{z}},-\frac{1}{z}\right) \\
E_{z}(z, \bar{z}) & \rightarrow \frac{1}{z^{2}} E_{\bar{z}}\left(-\frac{1}{\bar{z}},-\frac{1}{z}\right) \\
E_{\bar{z}}(z, \bar{z}) & \rightarrow \frac{1}{\bar{z}^{2}} E_{z}\left(-\frac{1}{\bar{z}},-\frac{1}{z}\right)
\end{aligned}
$$

Again we proceed to check the compatibility of the identifications under this new 
discrete symmetry, that is $t^{\prime} \cong 1-t, z^{\prime} \cong-1 / \bar{z}$ and $\bar{z}^{\prime} \cong-1 / z$. We obtain for $P_{2} C T$

$$
\begin{aligned}
P_{2} C T: \quad(t, z, \bar{z}) & =\Lambda\left(1-t,-\frac{1}{\bar{z}},-\frac{1}{z}\right) \\
\partial_{i} E^{i}(t, z, \bar{z}) & =-\frac{1}{z^{2} \bar{z}^{2}} \partial_{i} E^{i}\left(1-t,-\frac{1}{\bar{z}},-\frac{1}{z}\right) \\
B(t, z, \bar{z}) & =-\frac{1}{z^{2} \bar{z}^{2}} B\left(1-t,-\frac{1}{\bar{z}},-\frac{1}{z}\right) \\
Q(t)=\int_{\Sigma(t)} \rho_{0} & =Q(1-t)=-\int_{\tilde{\Sigma}(1-t)}\left(-\rho_{0}\right)
\end{aligned}
$$

Note that the relation between the integrals

$$
\int_{\tilde{\Sigma}\left(t^{\prime}\right)} \frac{d^{2} z^{\prime}}{z^{\prime 2} \bar{z}^{\prime 2}} z^{\prime 2} \bar{z}^{\prime 2}\left(B\left(t^{\prime}, z^{\prime}, \bar{z}^{\prime}\right)+\partial_{i} E^{i}\left(t^{\prime}, z^{\prime}, \bar{z}^{\prime}\right)\right)=\int_{\Sigma(t)} d^{2} z\left(B(t, z, \bar{z})+\partial_{i} E^{i}(t, z, \bar{z})\right)
$$

follows from taking into account the second and third equalities of (4.23), and the relations $d z=d \bar{z}^{\prime} / \bar{z}^{\prime 2}, d \bar{z}=d z^{\prime} / z^{\prime 2}$, and consequently $d z \wedge d \bar{z}=-\left(1 / z^{\prime} \bar{z}^{\prime}\right) d z^{\prime} \wedge d \bar{z}^{\prime}$. $\Sigma$ and $\tilde{\Sigma}$ again have opposite orientations and are mapped into each other by the referred involution. Under these relations and in a similar way to (4.24) the action transforms under $P_{2}$ as given in (4.16) and any of the combinations $P_{2} C T$ or $P_{2} T$ keep it invariant. Also the Wilson lines have the same property given by (4.20).

In the derivation of the previous identifications (4.23) we had to demand analyticity of the fields on the full sphere. This translates into demanding the transformation between the two charts covering the sphere to be well defined. Since $\partial_{u} \Lambda=-z^{2} \partial_{z} \Lambda$ and $\partial_{\bar{u}} \Lambda=-\bar{z}^{2} \partial_{\bar{z}} \Lambda$ the fields must behave at infinity and zero like

$$
\begin{aligned}
& \Lambda \stackrel{\infty}{\rightarrow} z^{-1} \bar{z}^{-1} \quad \Lambda \stackrel{0}{\rightarrow} z^{3} \bar{z}^{3} \\
& A_{z} \stackrel{\infty}{\rightarrow} z^{-2} \bar{z}^{-1} \quad A_{z} \stackrel{0}{\rightarrow} z^{2} \bar{z} \\
& A_{\bar{z}} \stackrel{\infty}{\rightarrow} z^{-1} \bar{z}^{-2} \quad A_{\bar{z}} \stackrel{0}{\rightarrow} z \bar{z}^{2}
\end{aligned}
$$

If naively we didn't care about these last limits the relations would be plagued with Dirac deltas coming from the identity $2 \pi \delta^{2}(z, \bar{z})=\partial_{z}(1 / \bar{z})=\partial_{\bar{z}}(1 / z)$. Once the previous behaviors are taken into account all these terms will vanish upon integration. Another way to interpret these results is to note that the points at infinity are not part of the chart (not physically meaningful), to check the physical behavior at those points we have to compute it at zero in the other chart.

This time the charges compatible with $P_{2} C T$ are $q=m$ since $q \cong \bar{q}$. Once there are no boundaries it is not possible to have configurations with two Wilson which allow this kind of orbifold. In this way the lowest number of lines is four as pictured in figure 16. Furthermore the number of Wilson lines must be even.

This configuration corresponds to untwisted closed unoriented string theories. Note that $\Lambda$, which is identified with string theory target space, is not orbifolded by $P_{2} C T$. The charges allowed are $q=m$, the KK momenta of string theory. Once again the monopole processes are suppressed. 


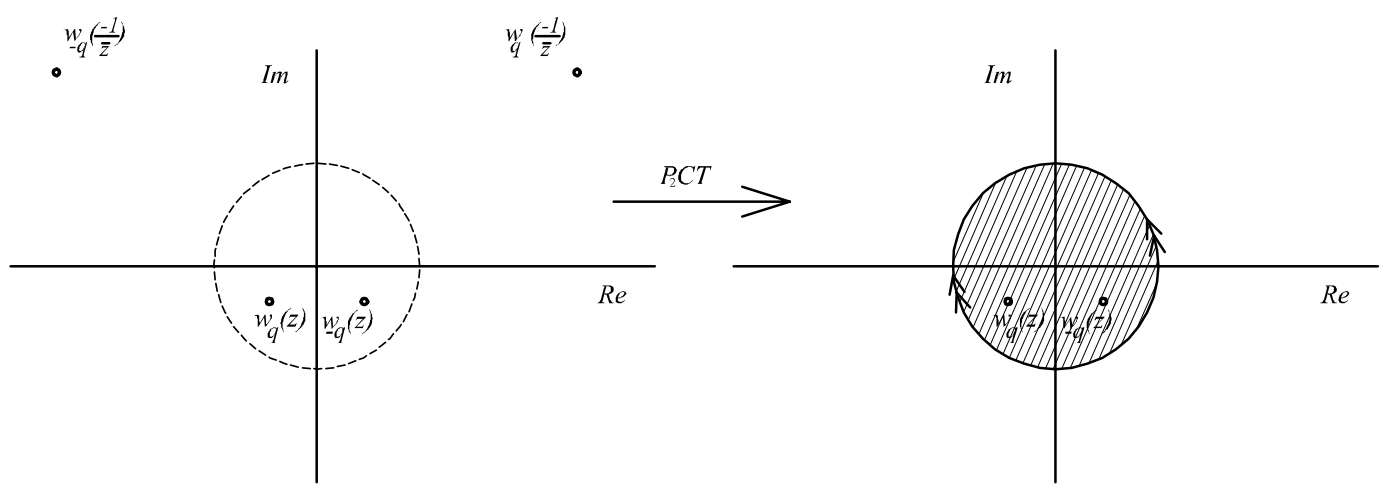

Figure 16: Orbifold under PCT in the presence of 4 Wilson lines, two $W_{q}$ and two $W_{-q}$. The new $2 d$ boundary is $\Sigma_{o \frac{1}{2}}=S^{2} / P=R P_{2}$. The allowed charges are $q=m$.

For $P_{2} T$ the fields relate as

$$
\begin{aligned}
& P_{2} T: \quad \Lambda(t, z, \bar{z}) \quad=\quad-\Lambda\left(1-t,-\frac{1}{\bar{z}},-\frac{1}{z}\right) \\
& \partial_{i} E^{i}(t, z, \bar{z})=\frac{1}{z^{2} \bar{z}^{2}} \partial_{i} E^{i}\left(1-t,-\frac{1}{\bar{z}},-\frac{1}{z}\right) \\
& B(t, z, \bar{z})=\frac{1}{z^{2} \bar{z}^{2}} B\left(1-t,-\frac{1}{\bar{z}},-\frac{1}{z}\right) \\
& Q(t)=\int_{\Sigma(t)} \rho_{0}=-Q(1-t)=-\int_{\tilde{\Sigma}(1-t)}\left(-\rho_{0}\right)
\end{aligned}
$$

In this case $q=k n / 4$ since $q \cong-\bar{q}$ and further configurations with two Wilson lines are compatible with the orbifold as pictured in figure 17 .
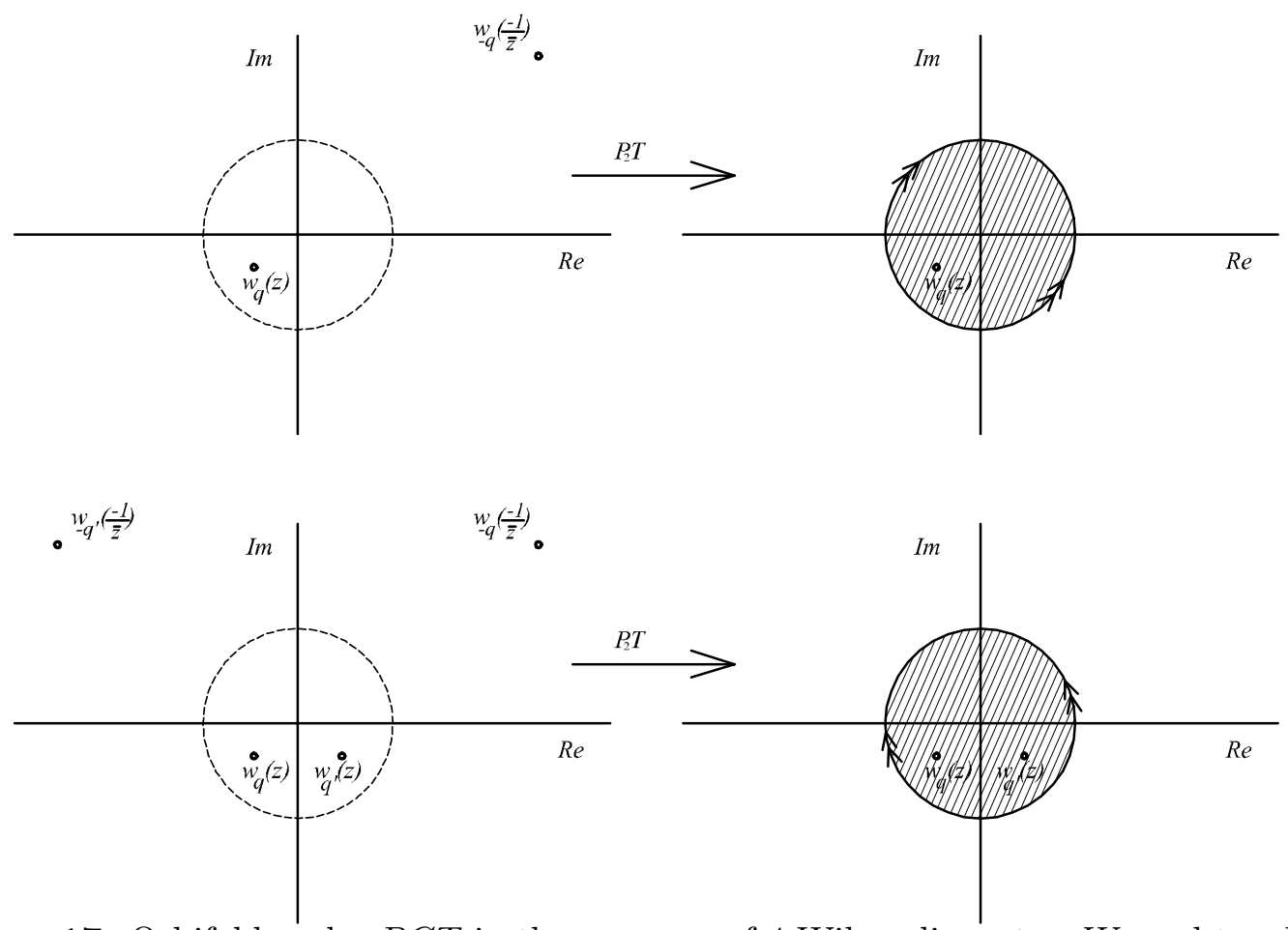

Figure 17: Orbifold under PCT in the presence of 4 Wilson lines, two $W_{q}$ and two $W_{-q}$. The new $2 d$ boundary is $\Sigma_{o \frac{1}{2}}=S^{2} / P=R P_{2}$. The allowed charges are $q=m$. 
In this case we have twisted unoriented closed strings. Note that the orbifold identifies $\Lambda \cong-\Lambda$ such that the target space of string theory is orbifolded. The full construction, including the world-sheet parity, from the point of view of string theory is called an orientifold. The allowed charges $q=k n / 4$ correspond to the winding number of string theory. The monopole processes are again crucial since allow, in the new boundary, the gluing of Wilson lines carrying opposite charges. We will return to this discussion.

\subsection{One Loop Amplitudes for Open and Closed Unoriented Strings}

One loop amplitudes are computed for Riemann surfaces of genus 1. They correspond to the torus (closed oriented) and its orbifolds: the annulus or cylinder (open oriented), the Möbius strip (open unoriented) and the Klein bottle (closed unoriented).

\subsubsection{Annulus}

We start with the already studied parity transformation $\Omega$, as given by (2.15). There is nothing new to add to the fields relations (4.19) for PCT and (4.21) for PT, this time under the identifications $t^{\prime}=1-t, z^{\prime}=-\bar{z}$ and $\bar{z}^{\prime}=-z$. The resulting geometry is the annulus $C_{2}$ and has now two boundaries.
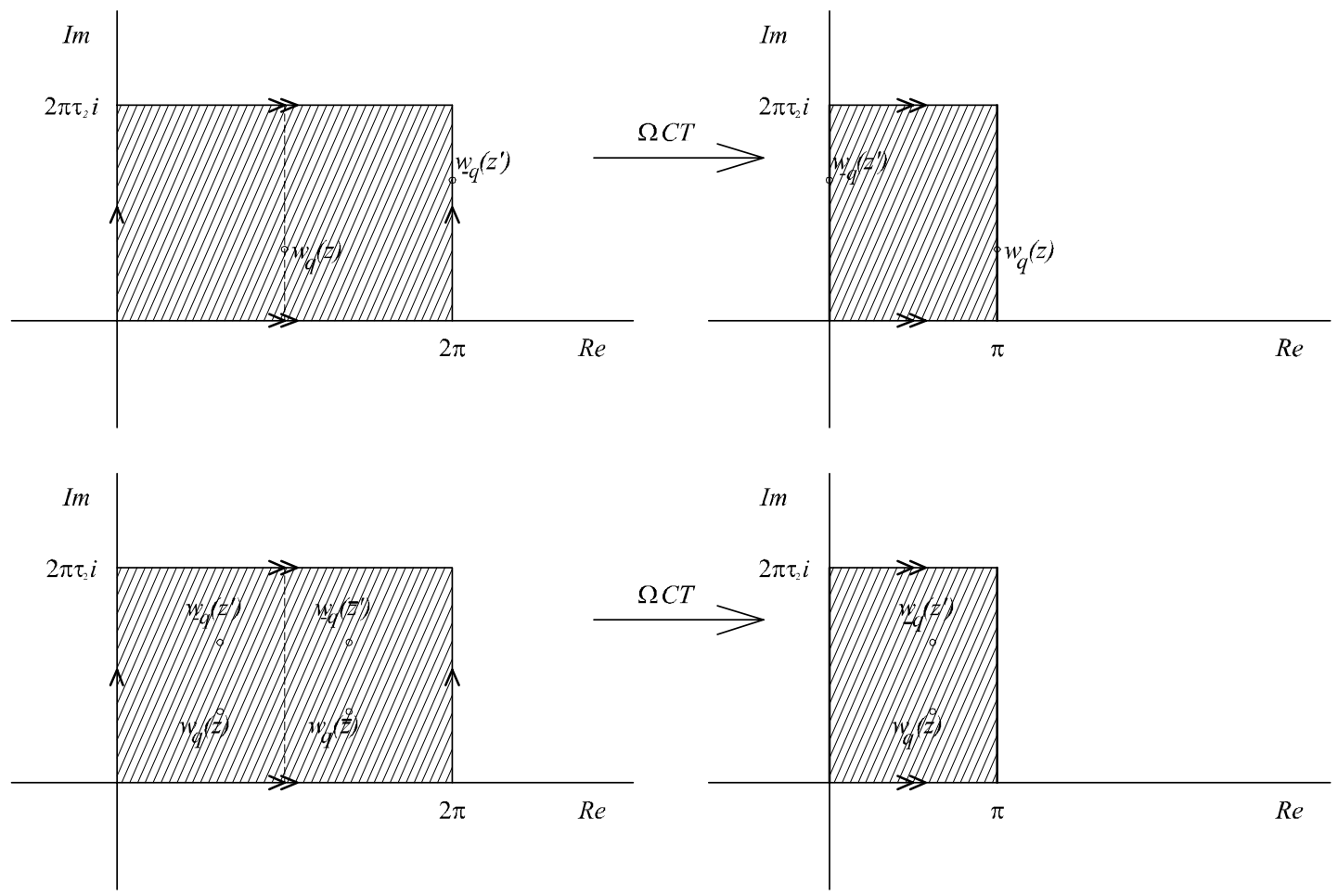

Figure 18: Orbifold under $\Omega C T$ in the presence of 2 and 4 Wilson lines. The new $2 d$ boundary is $\Sigma_{o \frac{1}{2}}=S^{2} / \Omega=C_{2}$. The allowed charges are $q=m$. 
For $\Omega C T$ the allowed charges are $q=m$ due to the identification $q \cong \bar{q}$ and $B(x)=0$ at the boundaries. We can have two insertions in the boundaries of the $2 d$ CFT but not in the bulk due to the identifications of charges, basically the argument is the same as used for the disk. As in the disk we cannot have one single bulk insertion due to the total charge being necessarily zero in the full plane. Up to configurations with four Wilson lines we can have: two insertions in the boundary; one insertion in the bulk and one in the boundary corresponding to three Wilson lines; three insertions in the boundaries (with $\sum q=0$ ); one insertion in the bulk and two in the boundary corresponding to four Wilson lines; and two insertions in the bulk corresponding to four Wilson lines as pictured in figure 18. This construction corresponds to open oriented strings with Neumann boundary conditions. The charge spectrum is $q=m$, corresponding to KK momenta in string theory and the monopole induced processes are suppressed. It is Neumann because the gauged symmetry is of PCT type. We note that the definition of parity is not important, even for genus 1 surfaces the results hold similarly to the previous cases for $P_{1}$ and $P_{2}$ used in genus 0 . What is important is the inclusion of the discrete symmetry $C$ !

For $\Omega T$ the allowed charges are $q=k n / 4$ due to the identification $q \cong-\bar{q}$. There are no insertions in the boundary. One insertion in the bulk corresponds to two Wilson lines and two to four Wilson lines presented in picture 19 .

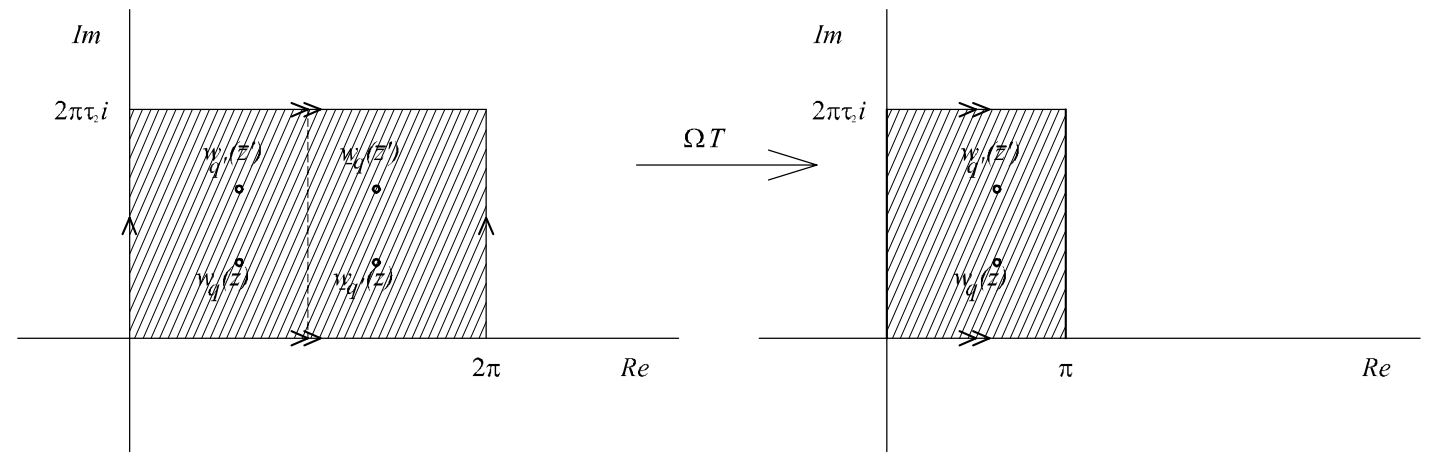

Figure 19: Orbifold under $\Omega T$ in the presence of 4 Wilson lines. The new $2 d$ boundary is $\Sigma_{o \frac{1}{2}}=S^{2} / \Omega=C_{2}$. The allowed charges are $q=k n / 4$.

This last construction corresponds to open oriented strings with Dirichlet boundary conditions. The charge spectrum is $q=k n / 4$, corresponding to the winding number in string theory, and the monopole induced processes are present allowing the gluing of Wilson lines with opposite charges.

\subsubsection{Möbius Strip}

Let us proceed to the parity $\tilde{\Omega}$ as given by (2.17). The results are pictured in figure 20 and are fairly similar. Note that it corresponds to two involutions of the torus with $\tau=2 i \tau$, one given by $\Omega$ resulting in the annulus, and $\tilde{a}$ which maps the annulus into the Möbius strip. Then, for each insertion in the strip it is necessary to exist four in the torus. 

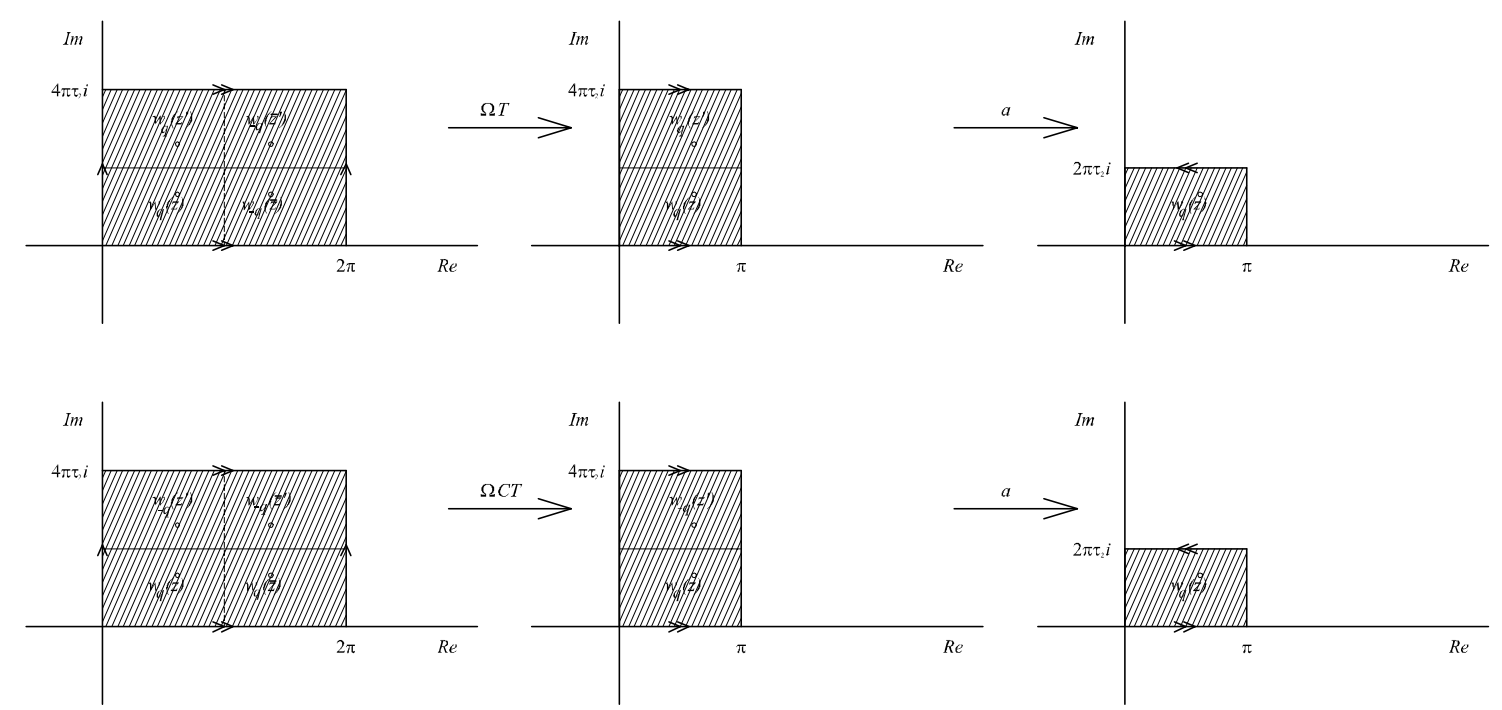

Figure 20: Orbifold under $\tilde{\Omega} T$ and $\tilde{\Omega} C T$ of the torus with $\tau=2 i \tau_{2}$ in the presence of four Wilson lines. For $\tilde{\Omega} C T$ the Wilson lines may pierce the manifold in the real axis and the allowed charge is $q=m$. For $\tilde{\Omega} T$ it is not allowed the existence of boundary insertions and the admissible charges are $q=k n / 4$. The relation $z^{\prime}=z+2 \pi\left(1 / 2+i \tau_{2}\right)$ must hold.

Once more we have for $\tilde{\Omega} C T$ that $B=-B=0$ in the boundaries and $q$ is identified with $\bar{q}$ demanding the charges to be $q=m$, which correspond to the KK momenta of string theory. Due to this fact the monopole processes are suppressed in the configurations allowing this kind of orbifolding. This corresponds to Neumann boundary conditions.

For the $\tilde{\Omega} T$ case we have the identification of $q$ with $-\bar{q}$ demanding the charges to be $q=k n / 4$, the winding number of string theory. This time not allowing the monopole processes to play an important role, the charges are purelly magnetic. This corresponds to Dirichlet boundary conditions.

As discussed in subsection 2.2 we can also consider the involution of the torus, with moduli $\tau=1 / 2+i \tau_{2}$ under $\Omega T$ or $\Omega C T$. In this case four insertions in the torus correspond to two insertions in the strip as presented in figure 21 for the $\Omega T$ case.

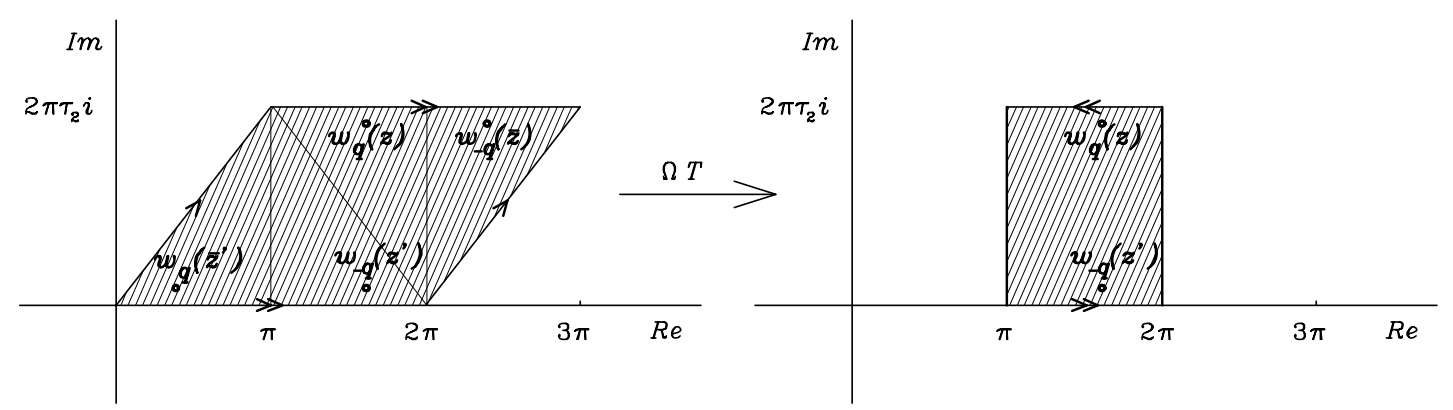

Figure 21: Orbifold under $\Omega T$ of the torus with $\tau=1 / 2+i \tau_{2}$ in the presence of four Wilson lines.

As previously explained both constructions result in the same region of the com- 
plex plane. Note that the resulting area in both cases is $2 \pi^{2} \tau_{2}$ and that in both cases the region $[0, \pi] \times i\left[0,2 \pi \tau_{2}\right]$ is identified with the region $[\pi, 2 \pi] \times i\left[0,2 \pi \tau_{2}\right]$.

\subsubsection{Klein Bottle}

Finally using the parity $\Omega^{\prime}$ as given by (2.19), we identify points under $t^{\prime}=1-t$, $z^{\prime}=-\bar{z}+2 \pi i \tau_{2}$ and $\bar{z}^{\prime}=-z+2 \pi i \tau_{2}$. Upon orbifolding the new boundary of TM is a Klein bottle.

Again, for $\Omega^{\prime} C T$, we obtain $q=m$ because $q \cong \bar{q}$. The minimum number of insertions is two corresponding to four Wilson lines in the bulk. This construction corresponds to untwisted unoriented closed strings with only KK momenta in the spectrum. The monopole processes are suppressed.

For $\Omega^{\prime} T$ case we have $q=k n / 4$ due to $q \cong-\bar{q}$. We can have one single insertion in the bulk corresponding to two Wilson lines or two corresponding to four Wilson lines. This construction corresponds to twisted unoriented closed strings with only winding number. The monopole processes are present and are crucial in the construction.
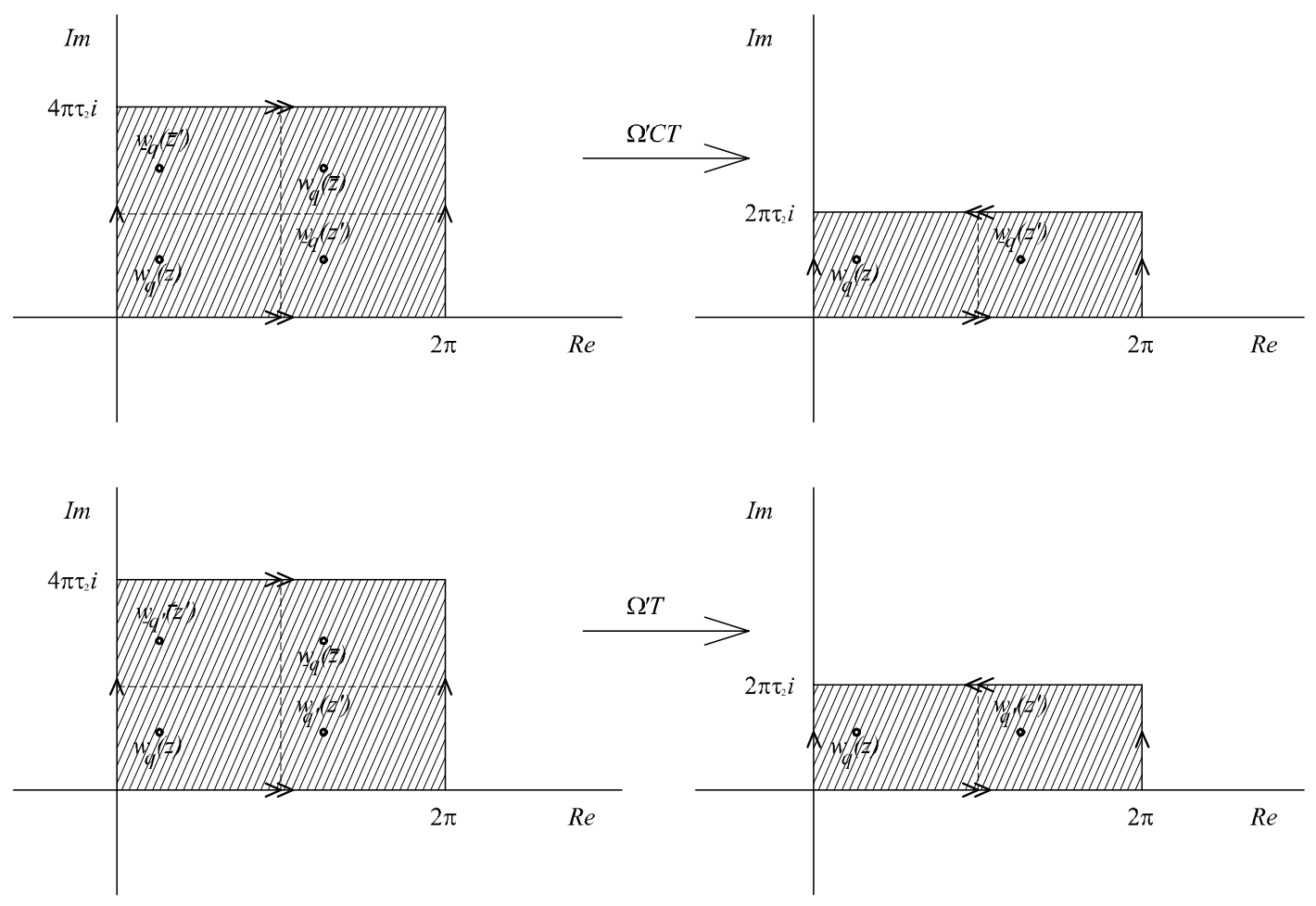

Figure 22: Orbifold of $\Sigma_{\frac{1}{2}}=T^{2}$ under $\Omega^{\prime} C T$ and $\Omega^{\prime} T$.

Two examples corresponding to four Wilson lines are pictured in figure 22.

\subsection{Note on Modular Invariance and the Relative Modular Group}

Modular invariance is a fundamental ingredient in string theory which makes closed string theories UV finite. What about the orbifolded theories? It is much more tricky. 
Each separated sector of open and unoriented theories is clearly not invariant under a modular transformation. The transformation $\tau \rightarrow-1 / \tau$ can be interpreted as the exchange of the holonomy cycles $\alpha$ and $\beta$ of the torus as represented in figure 23 . Equivalently it swaps $P T$ and PCT orbifold types. But then, if the first orbifold corresponds to the twisted sector (closed unoriented strings) or to Dirichlet boundary conditions (open strings), the second orbifold will correspond to the untwisted sector (closed unoriented strings) or to Dirichlet boundary conditions (open strings). Note the sign of the charges in figure 23 representing the Klein bottle projection.
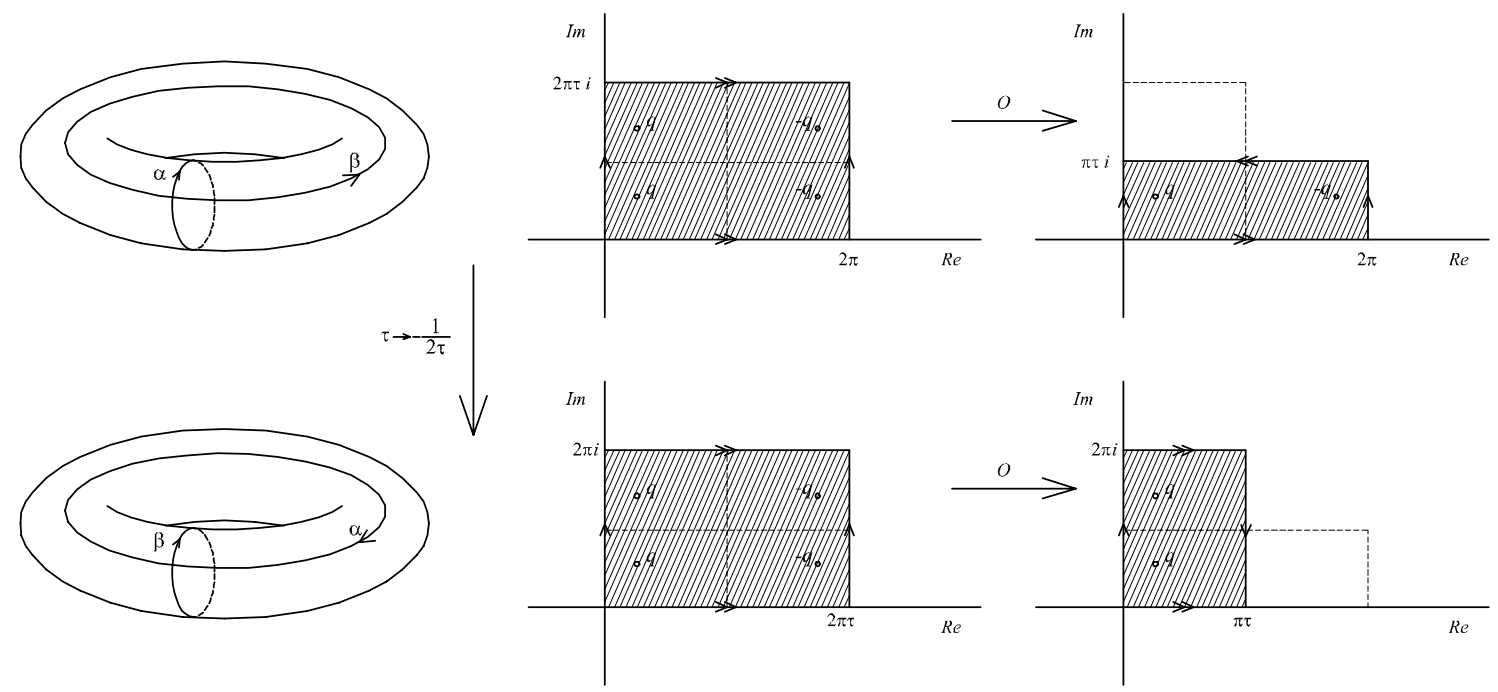

Figure 23: A suitable modular transformation takes us from one projection $P T$ (orbifold) to the other one $P C T$.

So if we actually want to ensure modular invariance we need to build a projection operator which ensures it. A good choice would be

$$
O=\frac{2+P T+P C T}{4}
$$

such that the exchange of orbifolds doesn't change it. This fact is well known in string theory (see [24] for details).

In the case when we are dealing with orbifolds which result in open surfaces the modular transformation $\tau \rightarrow-1 / \tau$, according to the previous discussion, exchanges the boundary conditions (Neumman/Dirichlet). Note that orbifolding the target space in string theory (or equivalently the gauge group in TMGT) is effectively creating an orientifold plane where the boundary conditions must be Dirichlet (as for a D-brane). This is the equivalent of twisting for open strings. In terms of the bulk the modular transformation is exchanging the projections $P C T \leftrightarrow P T$.

Let us put it in more exact terms. Consider some discrete group $H$ of symmetries of the target space (or equivalently the gauge group of TMGT). Consider now the 

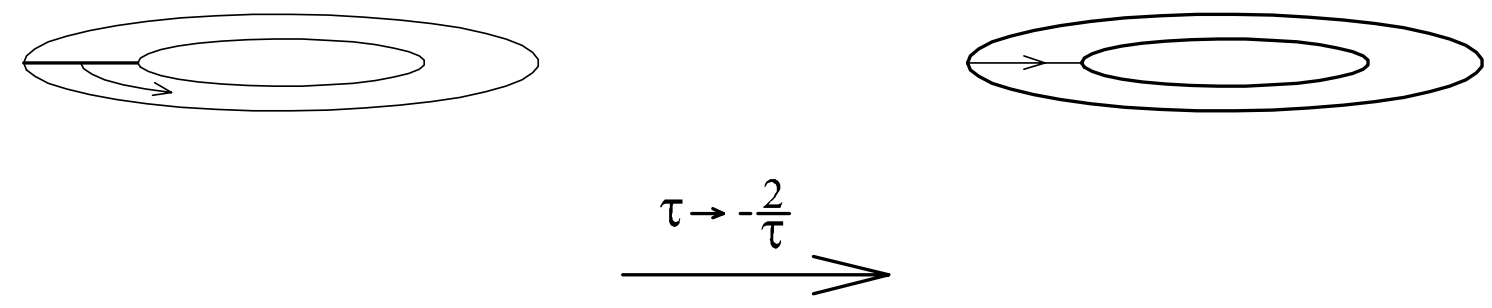

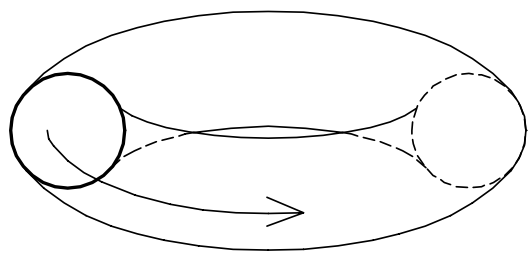

\section{Direct-Channel}

open loop

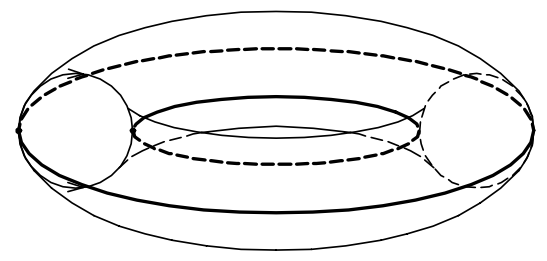

Transverse-Channel

close boundary to boundary

Figure 24: A suitable modular transformation takes us from the direct-channel picture to the transverse-channel picture. Here we show this construction in terms of the thickened string

twist by the element $h=\left(h_{1}, h_{2}\right) \in H$, where $h_{1}$ twists the states in the $x_{1}$ direction and $h_{2}$ in the $x_{2}$ direction. Then the modular transformation will change the twist as

$$
\begin{aligned}
& \mathcal{T}: \tau \rightarrow \tau+1\left(h_{1}, h_{2}\right) \rightarrow\left(h_{1}, h_{1} h_{2}\right) \\
& \mathcal{S}: \tau \rightarrow-\frac{1}{\tau} \quad\left(h_{1}, h_{2}\right) \rightarrow\left(h_{2}, h_{1}^{-1}\right)
\end{aligned}
$$

Returning to the Horava picture of describing an open string as a thickened surface (or double cover), in the case of the orbifold resulting in a new open boundary the picture is similar. In this case a modular transformation takes a open string loop, with the ends attached to the boundaries (direct-channel picture) to a closed string propagating from boundary to boundary (transverse-channel picture) as pictured in figure 24 for the annulus.

The lower boundary of the membrane is a thickening of the string. In the case of the open string loop we can think that the open string, while propagating, splits into two parts. The left modes propagate in the top half of the torus while the right modes propagate in the bottom half of the torus. In the case of the closed string, we again have a splitting of the closed string exactly as before but the propagation of the modes is transverse to the previous case as pictured in figure 24 .

Basically this discussion explains relations (4.10). The direct channel-picture on the disk correspond to $\operatorname{Tr}_{\text {open }}\left(e^{-H_{o} \tau}\right)$ where the trace is considered over the possible Chan-Paton factors carried by the open string. The transverse-channel picture corresponds to $\left\langle B\left|e^{-H_{o} \tilde{\tau}}\right| B\right\rangle$ where $|B\rangle$ stands for the states of the closed 
string.

So far we have concentrated on one loop amplitudes only, i.e. genus 1 world-sheet surfaces orbifolds. For the pure bosonic case this is sufficient, but once we introduce fermions and supersymmetry new constraints emerge at two loop amplitudes. Specifically the modular group of closed Riemann surfaces at genus $g$ is $S L(2 g, \mathbb{Z})$, upon orbifolding there is a residual conformal group, the so called Relative Modular Group [18] (see also [19-21]). For genus 1 this group is trivial but for higher genus it basically mixes neighboring tori, this means it mixes holes and crosscaps (note that any surface of higher genus can be obtained from sewing genus 1 surfaces). Furthermore, the string amplitudes defined on these genus 2 open/unoriented surfaces must factorise into products of genus 1 amplitudes. For instance a 2 torus amplitude can be thought as two 1 torus amplitudes connected trough an open string. For a discussion of the same kind of constraints for closed string amplitudes see [12-16].

The factorization and modular invariance of open/unoriented superstring theories amplitudes will induce generalized GSO projections ensuring the consistency of the resulting string theories.

The correct Neveu-Schwarz (NS - antiperiodic conditions, target spacetime fermions) and Ramond ( $\mathrm{R}$ - periodic conditions, target spacetime bosons) sectors were built from TMGT in [36]. There the minimal model given by the coset $M_{k}=S U(2)_{k+2} \times$ $S O(2)_{2} / U(1)_{k+2}$ with the CS action

$$
S^{N=2}[A, B, C]=k S_{C} S^{S U(2)}[A]+2 S_{C} S^{S O(2)}[B]-(k+2) S_{C} S^{U(1)}[C]
$$

was considered. It induces, on the boundary, an $N=2$ Super Conformal Field Theory (see also [33] for $N=1$ SCFT). The boundary states of the $3 D$ theory corresponding to the NS and R sectors are obtained as quantum superpositions of the 4 possible ground states (wave functions corresponding to the first Landau level the ground state is degenerate) of the gauge field $B$, that is to say we need to choose the correct basis of states. The GSO projections emerge in this way as some particular superposition of those 4 states at each boundary (for further details see [36]). It still remains to see how these constraints emerge from genus 2 amplitudes from TM and its orbifolds. We will discuss in detail these topics in some other occasion.

\subsection{Neumann and Dirichlet World-Sheet Boundary Conditions, Monopoles Processes and Charge Conjugation}

It is clear by now that the operation of charge conjugation $C$ is selecting important properties of the new gauged theory. And here we are referring to the properties of the $2 D$ boundary string theory. Gauging PCT results in having an open CFT with Neumann boundary conditions while, gauging $P T$ results in having Dirichlet boundary conditions. So $C$ effectively selects the kind of boundary conditions! In the case that PCT gives a closed unoriented manifold, we obtain an untwisted theory, 
while $P T$ gives a twisted theory (orientifold $X \cong-X$ ). Again $C$ effectively selects the theory to be twisted or not. These results are summarized in table 2 .

\begin{tabular}{|c|c|c|c|c|c|c|}
\hline & $P_{1}$ & $P_{2}$ & & $\Omega$ & $\tilde{\Omega}$ & $\Omega^{\prime}$ \\
\hline$S^{2} \rightarrow$ & $\begin{array}{c}D_{2} \\
\mathrm{O} / \mathrm{O}\end{array}$ & $\begin{array}{l}R P_{2} \\
\mathrm{C} / \mathrm{U}\end{array}$ & $T^{2} \rightarrow$ & $\begin{array}{c}C_{2} \\
\mathrm{O} / \mathrm{O}\end{array}$ & $\begin{array}{c}M_{2} \\
\mathrm{O} / \mathrm{U}\end{array}$ & $\begin{array}{c}K_{2} \\
\mathrm{C} / \mathrm{U}\end{array}$ \\
\hline$C T$ & $\begin{array}{c}N \\
\mathrm{q}=\mathrm{m}\end{array}$ & $\begin{array}{c}\text { Untwisted } \\
\qquad \mathrm{q}=\mathrm{m}\end{array}$ & & $\begin{array}{c}N \\
q=m\end{array}$ & $\begin{array}{c}N \\
q=m\end{array}$ & $\begin{array}{c}\text { Untwisted } \\
\qquad \mathrm{q}=\mathrm{m}\end{array}$ \\
\hline$T$ & $\begin{array}{c}D \\
q=k n / 4\end{array}$ & $\begin{array}{c}\text { Twisted } \\
q=k n / 4\end{array}$ & & $\begin{array}{c}D \\
q=k n / 4\end{array}$ & $\begin{array}{c}D \\
q=k n / 4\end{array}$ & $\begin{array}{c}\text { Twisted } \\
q=k n / 4\end{array}$ \\
\hline
\end{tabular}

Table 2: Boundary conditions and twisted sectors.

Although these facts are closely related with strings T-duality, the $C$ operation does not give us the dual spectrum. Upon gauging the full theory it is only selecting the Kaluza-Klein momenta or winding number as the spectrum of the configurations being gauged.

From the point of view of the bulk theory the gauged configurations corresponding to Neumann boundary conditions correspond to two Wilson lines with one end attached to the $1 D$ boundary of the new $2 D$ boundary of the membrane at $t=1 / 2$ and the other end attach to the $2 D$ boundary at $t=0$. For Dirichlet boundary conditions there is one single Wilson line with both ends in the $2 D$ membrane boundary at $t=0$ and a monopole insertion in the bulk of the $2 D$ boundary at $t=1 / 2$. Note that the Wilson lines do not, any longer, have a well defined direction in time, we have gauged time inversion. These results are presented in figure 25.

For the case where we get unoriented manifolds the picture is quite similar. There are always an even number of bulk insertions. In the case of PCT the Wilson lines which are identified have the same charge, therefore there are no monopole processes involved. The two Wilson lines are glued at $t=1 / 2$ becoming in the orbifolded theory one single line which has both ends attached to $\Sigma_{0}$ and one point in the middle belonging to $\Sigma_{1 / 2}$. In the boundary CFT we see two vertex insertions with opposite momenta. This construction corresponds to untwisted string theories since the target space coordinates (corresponding to the gauge parameter $\Lambda$ in $\mathrm{TM}(\mathrm{GT})$ ) are not orbifolded.

In the case of PT the identification is done between charges of opposite signs. Then two Wilson lines become one single line with its ends attached to $\Sigma_{0}$, but at one end they have a $q$ charge and in the other end they have a $-q$ charge. In $\Sigma_{1 / 2}$ there is a monopole insertion which exchanges the sign of the charge. This 

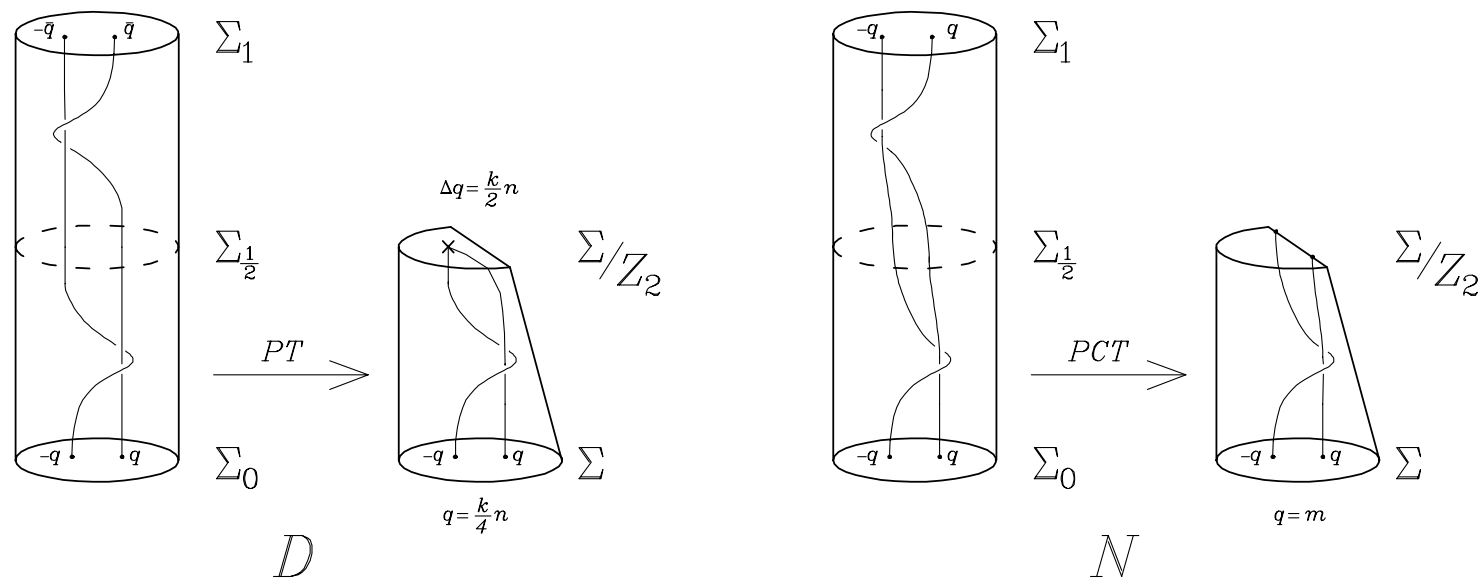

Figure 25: For Dirichlet boundary conditions two Wilson lines carrying charges $q$ and $-q$ meet in a monopole at the orbifold singular point $t=1 / 2$. While for Neumann boundary conditions the two Wilson lines end in the boundary of $\Sigma / Z_{2}$.

construction corresponds to twisted string theories since the target space coordinates are orbifolded $(\Lambda \cong-\Lambda)$.

As a final consistency check in PCT the charges are always restricted to be $q=m$ due to compatibility with the orbifold construction. By restricting the spectrum to these form we are actually eliminating the monopole processes for this particular configurations!

\subsection{T-Duality and Several U(1)'s}

The well know Target space or T-duality(for a review see [53]) of string theory is a combined symmetry of the background and the spectrum of momenta and winding modes. It interchanges winding modes with Kaluza-Klein modes. From the point of view of the orbifolded $\mathrm{TM}(\mathrm{GT})$ corresponding to open and unoriented string theories the projections $P T$ truncate the charges spectrum to $q=k n / 4$ (due to demanding $q=-\bar{q}$ ) which in string theory is the winding number. The projections $P C T$ truncate the charge spectrum to $q=m$ (due to demanding $q=\bar{q}$ ) which corresponds in string theory to the KK momenta. Note that PCT excludes all the monopole induced processes while $P T$ singles out only monopole induced processes $[32,37,39]$.

T-duality is, from the point of view of the $3 D$ theory, effectively exchanging the two kinds of projections

$$
\begin{aligned}
& \text { T-duality : } P T \leftrightarrow P C T \\
& q=-\bar{q} \leftrightarrow q=\bar{q}
\end{aligned}
$$

This is precisely what it must do. The nature of duality in $3 D$ terms was discussed in some detail in [35]. It was shown there that it exchange topologically non 
trivial matter field configurations with topologically non trivial gauge field configurations. Although charge conjugation was not discussed there (only parity and time inversion), this mechanism can be thought as a charge conjugation operation. Note that $C^{2}=1$.

It is also rather interesting that from the point of view of the membrane both T-duality and modular transformations are playing the same role. In some sense both phenomena are linked by the $3 D$ bulk theory.

So far we have considered only a single compact $U(1)$ gauge group. But new phenomena emerge in the more general case. The extra gauge sectors are necessary any how [39].

Take then the general action with gauge group $U(1)^{d} \times U(1)^{D}$ with $d U(1)^{\prime}$ s noncompact and the remaining $D$ 's compact.

$S_{d+D}=\int_{M} d x^{3}\left[-\frac{\sqrt{-g}}{\gamma}\left(F_{\mu \nu}^{M} F_{M}^{\mu \nu}+F_{\mu \nu}^{I} F_{I}^{\mu \nu}\right)+\frac{\epsilon^{\mu \nu \lambda}}{8 \pi}\left(K_{M N} A_{\mu}^{M} \partial_{\nu} A_{\lambda}^{N}+K_{I J} A_{\mu}^{I} \partial_{\nu} A_{\lambda}^{J}\right)\right]$

where $M, N=0, \ldots, d-1$ correspond to the non compact gauge group and $I, J=$ $d, \ldots, d+D-1$ to the compact ones.

For a given parity $P$ we can now build an operator $O$ that acts in every $A$ field through $P T$ and only in some of them through $C$

$$
O=P T\left(\sum_{I^{\prime}} C \delta_{I^{\prime} I}+\sum_{I^{\prime \prime}} \delta_{I^{\prime \prime} I}\right)
$$

Due to the charges not being quantize and the non existence of monopole-induced processes in the non compact gauge sector, the mechanism is slightly different (see section (3). But this operator can act as well over the noncompact sector.

For the case of open manifolds $M / P T, I^{\prime}$ run over the indices for which we want to impose Neumann boundary conditions (on $\Lambda^{I^{\prime}}$ ) and $I^{\prime \prime}$ over the indices corresponding to Dirichlet boundary conditions. For the case of closed manifolds $M / P T$ the picture is similar but $I^{\prime}$ runs over the indices we want $\Lambda^{I^{\prime}}$ to be orbifolded (obtaining an orientifold or twisted sector).

In the case of several $U(1)$ 's more general symmetries (therefore orbifold groups) can be considered (for instance $Z_{N}$ ). Those symmetries are encoded in the ChernSimons coefficient $K_{I J}$.

\section{Conclusion and Discussion}

In this paper we have shown how one can get open and closed unoriented string theories from the Topological Membrane. There were two major ingredients: one is the Horava idea about orbifolding, the second is that the orbifold symmetry was

a discrete symmetry of TMGT. The orbifold works from the point of view of the 
membrane as a projection of field configurations obeying either PT or PCT symmetries (the only two kinds of discrete symmetries compatible with TMGT). For PCT type projections we obtained Neumann boundary conditions for open strings and untwisted sectors for closed unoriented strings. For PT type projections we obtained Dirichlet boundary conditions for open strings and twisted sectors for closed unoriented strings. For $P C T q=\bar{q}=m$, so only the string Kaluza Klein modes survive. In this case the monopole induced processes are completely suppressed. For PT $q=-\bar{q}=k n / 4$, so only the string winding modes survive. In this case only monopole induced processes are present, being the charges purelly magnetic. Charge conjugation $C$ plays an important role in all the processes playing the role of a $Z_{2}$ symmetry of the string theory target space. These results can be generalized to symmetries of the target space encoded in the tensor $K_{I J}$ and are closely connected, both with modular transformations and T-duality which exchange $P T \leftrightarrow P C T$.

This work is the first part of our study of open and unoriented string theories. In the second part [54] we shall derive the partition functions of the boundary CFT from the bulk TMGT [55-59].

Also an important issue to address in future work will be to generalize the constructions presented here to non trivial boundary CFT's [54], for example WZNW models and different coset models which can be obtained from TM with non-Abelian TMGT.

As a final remark let us note that the string photon Wilson line has been left out. $\mathrm{TM}(\mathrm{GT})$ can take account of it as well: for any closed $\Sigma$ there is a symmetry of the gauge group coupling tensor $K_{I J} \rightarrow K_{I J}+\delta_{I} \chi_{J}-\delta_{J} \chi_{I}$ where each $\chi_{I}=\chi_{I}[A]$ is taken to be some function of the $A^{I}$ 's. This transformation affects only $B_{I J}$ and the induced terms vanish upon integration by parts. Once we consider the orbifold of the theory the new orbifolded $\Sigma_{o}$ has a boundary and the induced terms will not vanish any longer but induce a new action on the boundary $\partial \Sigma_{o}$, they will be precisely the new gauge photon action of open string theories. As is well known the choice of the gauge group of string theory, i.e. the Chan-Paton factors structure carried by this photon Wilson line will be determined by the cancellation of the open string theory gauge anomalies (see [24] and references therein). We postpone the proper treatment of this issue from the point of view of TM to another occasion [54].

\section{Acknowledgments}

The authors would like to thank Bayram Tekin for discussions and suggestions concerning discrete symmetries, Augusto Sagnotti for discussions and suggestions concerning modular invariance and to Alexander Nichols and Dave Skinner for reading the manuscript and helpful comments. PCF would also like to thank André Lukas and José Natário for useful discussions and suggestions concerning orbifold constructions and Nuno Reis for several discussions in random topics. The work of PCF is 
supported by PRAXIS XXI/BD/11461/97 grant from FCT (Portugal). The work of IK is supported by PPARC Grant PPA/G/0/1998/00567 and EUROGRID EU HPRN-CT-1999-00161.

\section{References}

[1] A. Sagnotti, Open Strings and their Symmetry Groups, talk presented at the Cargese Summer Institute on "Non-Perturbative Methods in Field Theory" (1987) 521-528

[2] C. P. Burgess and T. R. Morris, Nucl. Phys. B291 (1987) 256-284; Nucl. Phys. B291 (1987) 285-333

[3] J. P. Rodrigues, Phys. Lett. B178 (1986) 350-356; J. Math. Phys. 28 (1987) 2669

[4] J. A. Harvey and J. A. Minahan, Phys. Lett. B188 (1987) 44-50

[5] G. Pradisi and A. Sagnotti, Phys. Lett. B216 (1989) 59-67

[6] P. Horava, Nucl. Phys. B327 (1989) 461-484

[7] J. Govaerts, Phys. Lett. B220 (1989) 77

[8] P. Horava, Phys. Lett. B231 (1989) 251-257

[9] M. Bianchi, G. Pradisi and A. Sagnotti, Nucl. Phys. B376 (1992) 365-386

[10] M. Bianchi and A. Sagnotti, Nucl. Phys. B361 (1991) 519-538

[11] C. Angelantonj, M. Bianchi, G. Pradisi, A. Sagnotti and Ya. S. Stanev, Phys. Lett. B385 (1996) 96-102

[12] I. Antoniadis, C. P. Bachas and C. Kounnas, Nucl. Phys. B289 (1987) 87-108

[13] H. Kaway, D. C. Lewellen and S. H. H. Tye, Nucl. Phys. B288 (1987) 1

[14] W. Lerche, D. Lust and A. N. Schellekens, Nucl. Phys. B287 (1987) 477

[15] I. Antoniadis and C. P. Bachas, Nucl. Phys. B298 (1988) 586-612

[16] H. Kaway, D. C. Lewellen, J. A. Schwartz and S. H. H. Tye, Nucl. Phys. B299 (1988) 431

[17] P. Gliozzi, J. Scherk and D. Olive, Nucl.Phys. B122 (1977) 253-290

[18] M. Bianchi and A. Sagnotti, Phys. Lett. B231 (1989) 389-396

[19] M. Bianchi and A. Sagnotti, Phys. Lett. B211 (1988) 407-416

[20] A. Sagnotti, Phys. Rep. 184, Nos. 2-4 (1989) 167-175

[21] M. Bianchi and A. Sagnotti, Phys. Lett. B247 (1990) 517-524 
[22] A. Sagnotti, Talk Presented at SUSY 95, hep-th/9509080

[23] A. Sagnotti, Nucl. Phys. Proc. Suppl. 56B (1997) 332-343

[24] J. Polchinski, String Theory Cambridge Univ. Press

[25] I. I. Kogan, Phys. Lett. B231 (1989) 377

[26] S. Carlip and I. I. Kogan, Phys. Rev. Lett. 64 (1990) 1487; Phys. Rev. Lett. 67 (1991) 3647-3649

[27] S. Carlip and I. I. Kogan, Mod. Phys. Lett. A6, No 3 (1991) 171-181

[28] I. I. Kogan, Phys. Lett. B256 (1991) 369; Nucl. Phys. B375 (1992) 362

[29] S. Carlip, Nucl. Phys. B362 (1991) 111-124

[30] L. Cooper and I. I. Kogan, Phys. Lett. B383 (1996) 271-280

[31] G. Amelino-Camelia, I. I. Kogan and R. J. Szabo, Nucl. Phys. B480 (1996) 413-456; Int. J. Mod. Phys. A12 (1997) 1043-1052

[32] L. Cooper, I. I. Kogan and K.M. Lee, Phys.Lett. B394 (1997) 67-74

[33] I. Kogan, Phys. Lett. B390 (1997) 189-196

[34] L. Cooper, D.Phil Thesis, 1997

[35] L. Cooper, I. I. Kogan and R. J. Szabo, Annals. Phys. 268 (1998) 61-104

[36] L. Cooper, I. I. Kogan and R. J. Szabo, Nucl. Phys. B498 (1997) 492-510

[37] I. I. Kogan and R. J. Szabo, Nucl.Phys. B502 (1997) 383-418

[38] I. I. Kogan, A. Momen and R. J. Szabo, JHEP 9812 (1998) 013

[39] P. Castelo Ferreira, I. I. Kogan and Bayram Tekin, Nucl. Physics B589 (2000) 167-195

[40] For a review see Lectures on Topological Membranes in Campos do Jordão (1997) Particles and fields 223-290 http://www-thphys.physics.ox.ac.uk/users/IanKogan/membrane.ps

[41] W. Siegel, Nucl. Phys. B156 (1979) 135

[42] J. Schonfeld, Nucl. Phys. B185 (1981) 157

[43] S. Deser, R. Jackiw and S. Templeton, Phys. Rev. Lett. 48 (1982) 975, Ann. Phys. NY 140 (1982) 372

[44] E. Witten, Commun. Math. Phys. 121 (1989) 351-399

[45] G. Moore and N. Seiberg,Phys. Lett. B220 (1989) 422-430 
[46] S. Elitzur, G. Moore, A. Schwimmer and N. Seiberg, Nucl. Phys. B326 (1989) 108

[47] P. Horava, J. Geom. Phys. 21 (1996) 1-33

[48] L. Birke, J. Fuchs and C. Schweigert, Adv. Theor. Math. Phys. 3 (1999) 671-726

[49] G. Felder, J. Frolich, J. Fuchs and C. Schweigert, Phys. Rev. Lett. 84 (2000) 16591662; hep-th/9912239

[50] G. Dunne, Aspects of Chern-Simons Theory, Les Houches Lectures (1998), hepth/9902115

[51] J. L. Cardy, Nucl. Phys. B240[FS12] (1984) 514-532

[52] P. Di Francesco, P. Mathieu and D. Sénéchal, Conformal Field Theory, Springer

[53] A. Giveon,M. Porrati and E. Rabinovici, Phys.Rept. 244 (1994) 77-202

[54] Open and Unoriented Strings from Topological Membrane $\geq I I$, in preparation

[55] M. Bos and V. P. Nair, Phys. Lett. B223 (1989) 61; Int. J. Mod. Phys. A5 (1990) 959

[56] J. M. F. Labastida and A. V. Ramallo, Phys. Lett. B227 (1989) 92; B228 (1989) 214

[57] W. Ogura, Phys. Lett B229 (1989) 61

[58] M. Asorey, F. Falceto and S. Carlip, Phys. Lett. B312 (1993) 477-485

[59] E. Witten, Commun. Math. Phys. 144 (1992) 189-212 